Ergod. Th. \& Dynam. Sys. (1983), 3, 47-85

Printed in Great Britain

\title{
Rigidity of lattices of non-positive curvature
}

\author{
P. EBERLEIN \\ Department of Mathematics, University of North Carolina, Chapel Hill, North \\ Carolina 27514
}

(Received 26 October 1981)

Abstract. Let $M, M^{*}$ denote compact, connected manifolds of non-positive sectional curvature whose fundamental groups are isomorphic and whose Euclidean de Rham factors are trivial. We prove that: if $M$ is a compact irreducible quotient of a reducible symmetric space $H$, then $M$ and $M^{*}$ are isometric up to a constant multiple of the metric; and that the number and dimensions of the local de Rham factors are the same for $M$ and $M^{*}$. Gromov has independently proved the first result in the more general case that $M$ is locally symmetric and globally irreducible with rank at least two.

\section{0 . Introduction}

A basic problem in Riemannian geometry is to determine the extent to which the geometry of the Riemannian metric and the topology of the underlying manifold influence each other. Restrictions on the curvature and topology are usually necessary to obtain reasonable results, and we shall confine our attention to compact connected manifolds of nonpositive sectional curvature. We define a geometric property of such manifolds to be a rigid property if whenever it holds for a manifold $M$ it also holds for any manifold $M^{*}$ that is homotopically equivalent to $M$. Our goal is to look for rigid properties.

In a previous paper [7] we showed that certain geometric properties of a free homotopy class of closed curves are rigid properties. In this paper we have two main results, the first of which is half of an independent result of Gromov. Before stating them we define a Riemannian manifold $X$ to be reducible if some finite Riemannian cover $\tilde{X}$ splits as a nontrivial Riemannian product $\tilde{X}_{1} \times \tilde{X}_{2}$. If $X$ is simply connected and reducible, then $X$ itself is a nontrivial Riemannian product $X_{1} \times X_{2}$.

THEOREM A. Let $M, M^{*}$ denote compact connected Riemannian manifolds of nonpositive sectional curvature whose fundamental groups are isomorphic and whose universal Riemannian covering manifolds $H, H^{*}$ possess no Euclidean de Rham factor. Suppose that $H^{*}$ is a reducible symmetric space of noncompact type and $M^{*}$ is an irreducible quotient of $H^{*}$. Then $M$ and $M^{*}$ are isometric, provided that one multiplies the metric of $M$ or $M^{*}$ by a suitable positive constant. 
In particular if $\left(M^{*}, g^{*}\right)$ satisfies the hypotheses of theorem $A$, then the only metrics $g$ of nonpositive sectional curvature on the $C^{\infty}$ manifold $M^{*}$ are the constant multiples of $g^{*}$.

Our second result says that the fundamental group, a topological object, deterines the number and dimensions of the factors in the de Rham decomposition, a geometric property. At present we must exclude consideration of manifolds that have non-trivial Euclidean de Rham factors, but this restriction is probably unnecessary.

TheOReM B. Let $M, M^{*}$ denote compact, connected Riemannian manifolds of non-positive sectional curvature whose fundamental groups are isomorphic and whose universal Riemannian covering manifolds $H, H^{*}$ possess no Euclidean de Rham factor. Let $H^{*}=H_{1}^{*} \times \cdots \times H_{k}^{*}$ and $H=H_{1} \times \cdots \times H_{i}$ denote the de Rham decompositions of $H^{*}$ and $H$ with the factors ordered so that

$$
\operatorname{dim} H_{i}^{*} \leq \operatorname{dim} H_{i+1}^{*} \quad \text { for } 1 \leq i \leq k-1
$$

and

$$
\operatorname{dim} H_{r} \leq \operatorname{dim} H_{r+1} \quad \text { for } 1 \leq r \leq j-1 .
$$

Then

$$
j=k \text { and } \operatorname{dim} H_{i}^{*}=\operatorname{dim} H_{i} \quad \text { for all } 1 \leq i \leq k .
$$

(The case that $j=1$ or $k=1$ is allowed.)

Using Tits complexes Gromov [11] has proved independently the following result that includes our Theorem A:

THEOREM. Let $M, M^{*}$ be connected compact Riemannian manifolds of non-positive sectional curvature whose fundamental groups are isomorphic. Let $M^{*}$ be an irreducible locally symmetric manifold of rank $r \geq 2$. Then $M$ and $M^{*}$ are isometric, provided that one multiplies the metric of $M$ or $M^{*}$ by a suitable positive constant.

Gromov considers separately the cases where $M^{*}$ is locally reducible or locally irreducible. The first of these cases is our theorem A except that no exclusion of Euclidean de Rham factors is required. Our proof of theorem A avoids the use of Tits complexes although the 'maximally singular' geodesics that we consider are essentially the 1-simplices of the Tits complex associated to a Riemannian symmetric space of noncompact type.

The condition that the rank of $M^{*}$ in the theorem above be at least two is clearly necessary. A rank 1 locally symmetric space $\left(M^{*}, g^{*}\right)$ has strictly negative sectional curvature and there are obviously many nearby metrics $g$ of strictly negative sectional curvature on $M^{*}$ that are not locally symmetric. At the same time the theorem seems very plausible on intuitive grounds if the rank of $M^{*}$ is at least 2 . A locally symmetric space $\left(M^{*}, g^{*}\right)$ of rank $r \geq 2$ has through every point a large collection of totally geodesic immersed Euclidean spaces of dimension $r$. With so much zero sectional curvature present it is unlikely that one could perturb the metric $g^{*}$ to obtain a nearby metric $g$ of non-positive sectional curvature. If local 
rigidity of the metric $g^{*}$ is plausible, then global rigidity of $g^{*}$ as expressed in theorem A or the result of Gromov is not surprising.

We now outline briefly the proofs of theorems A and B. Theorem B follows fairly simply from theorem A, a Riemannian splitting theorem of $[10]$ or [16] and some results from [2], [6] on the structure of isometry groups. The full argument, which is short, may be found in $\S 6$. The proof is by induction on the dimension of $M$ and $M^{*}$.

The proof of theorem $A$ is much longer, though the basic idea, which we now outline, is not difficult. Clearly we wish to apply the Mostow rigidity theorem by showing that the manifold $H$, using the notation of theorem $\mathrm{A}$, is a symmetric space of noncompact type. Using [6, prop. 4.6] it will follow that $H$ is a symmetric space of noncompact type if we can show that $H$ is reducible. The method of the proof is first to construct a collection $\left\{B_{p}: p \in H\right\}$ of closed convex subsets of $H$ that are candidates for the leaves of a parallel foliation of $H$ and second to prove that these sets $\left\{B_{p}: p \in H\right\}$ actually are the leaves of a proper parallel foliation of $H$. These arguments are described in $\S 5$ and Appendix 2 and are very similar to those used to prove theorem 2 of [10]. Once we have a proper parallel foliation of $H$ it follows by the de Rham decomposition theorem that $H$ is reducible.

We now outline the proof of theorem A in somewhat greater detail. Let $M, M^{*}$, $H$ and $H^{*}$ be as defined in the statement of theorem A. The first step in the proof is to consider the 'maximally singular' geodesics of $H^{*}$, a symmetric space of noncompact type. A geodesic $\gamma^{*}$ of $H^{*}$ is said to be maximally singular if $\gamma^{*}(\mathbb{R})$ is the intersection of all $r$-flats of $H^{*}$ that contain $\gamma^{*}$. Here $r$ is the rank of $H^{*}$ and an $r$-flat is an $r$-dimensional, flat, totally geodesic, closed, imbedded submanifold of $H^{*}$. A point $x^{*} \in H^{*}(\infty)$ is said to be maximally singular if one (hence all) of its representative geodesics is maximally singular.

Suppose now that $H^{*}$ is the Riemannian product $H_{1}^{*} \times H_{2}^{*}$ of two symmetric spaces of noncompact type and positive dimension. The sets $H_{1}^{*}(\infty)$ and $H_{2}^{*}(\infty)$ are naturally imbedded as subsets of $H^{*}(\infty)$. Now let $N_{1}^{*}, N_{2}^{*}$ denote the orthogonal parallel foliations of $H^{*}$ induced by $H_{1}^{*}, H_{2}^{*}$. If $S_{i}^{*}$ denotes the set of maximally singular points in $H_{i}^{*}(\infty)$ for $i=1,2$, then one can show that

$$
N_{i}^{*}(p)=\operatorname{span}\left\{\gamma_{p x}^{\prime}(0): x \in S_{i}^{*}\right\}
$$

where $\gamma_{p x}$ denotes the geodesic starting at $p$ that belongs to the asymptote class $x$. Moreover the leaf of $N_{i}^{*}$ through $p$ is the smallest closed convex subset of $H^{*}$ that contains all maximal geodesics $\gamma_{p x}(\mathbb{R})$ as $x$ ranges over $S_{i}^{*}$ for $i=1,2$.

Now let $\Gamma^{*}, \Gamma$ be groups of isometries of $H^{*}, H$ such that $M^{*}=H^{*} / \Gamma^{*}$ and $M=H / \Gamma$. By hypothesis there exists an isomorphism $\theta: \Gamma^{*} \rightarrow \Gamma$. As in [17] we construct a $\theta$-equivariant pseudoisometry $f: H^{*} \rightarrow H$, that is, a uniformly continuous function $f$ such that:

(1) $f\left(\phi^{*} p^{*}\right)=\theta\left(\phi^{*}\right) f\left(p^{*}\right) \quad$ for all $p^{*} \in H^{*}, \phi^{*} \in \Gamma^{*}$,

(2) there exist positive constants $\xi^{*}, r^{*}$ such that

$$
\left(1 / \xi^{*}\right) d\left(p^{*}, q^{*}\right) \leq d\left(f p^{*}, f q^{*}\right) \leq \xi^{*} d\left(p^{*}, q^{*}\right)
$$

whenever $d\left(p^{*}, q^{*}\right) \geq r^{*}$. 
In $\S 4$ we show that $f$ determines a $\theta$-equivariant map $\bar{f}: S^{*} \rightarrow H(\infty)$, where $S^{*}$ denotes the set of maximally singular points in $H^{*}(\infty)$. Specifically if $\gamma^{*}$ represents $x^{*} \in S^{*}$ we show that $\left(f \circ \gamma^{*}\right)(t)$ converges to a point $\bar{f}\left(x^{*}\right) \in H(\infty)$ as $t \rightarrow+\infty$. The construction of the map $\bar{f}$ is valid in the general case that $H^{*}$ is an arbitrary symmetric space of noncompact type, not necessarily reducible.

Returning to the situation of theorem $\mathrm{A}$, we have represented $H^{*}$ as a non-trivial Riemannian product $H_{1}^{*} \times H_{2}^{*}$. For $i=1,2$ we define $S_{i}$ to be the closure in $H(\infty)$ of the set $\bar{f}\left(S_{i}^{*}\right)$, where $S_{i}^{*}$ is the set of all maximally singular points of $H_{i}^{*}(\infty)$. (Actually one may show that $\bar{f}$ is continuous and $\bar{f}\left(S_{i}^{*}\right)$ is already closed.) We regard the sets $S_{1}, S_{2}$ as candidates for the sets of maximally singular points of $H_{1}(\infty), H_{2}(\infty)$, where $H_{1} \times H_{2}$ is a Riemannian splitting of $H$ that is yet to be determined.

Working backwards from the disclission above we use the sets $S_{1}, S_{2}$ to construct a splitting $H=H_{1} \times H_{2}$. For each point $p \in H$ we define $B_{p}$ to be the smallest closed convex subset of $H$ that contains all geodesics $\gamma_{p x}(\mathbb{R})$ as $x$ ranges over $S_{1}$. In $\S 5$ we show that the sets $B_{p}$ are proper, complete, totally geodesic submanifolds of $H$ that are the leaves of a parallel foliation $N_{1}$ of $H$. If $Q_{p}$ denotes the leaf through $p$ of the orthogonal parallel foliation $N_{2}$ then $H$ is isometric to the Riemannian product of $B_{p}$ and $Q_{p}$, by the de Rham decomposition theorem. This completes the outline of theorem $A$.

We conclude the introduction with a brief description of the organization of the paper. The first section contains preliminary material. Using the notation of theorems $\mathrm{A}$ and $\mathrm{B}$ we define and discuss the properties of a pseudoisometry $f: H^{*} \rightarrow H$ that is induced by an isomorphism of the fundamental groups of $M^{*}$ and $M$. In $\S 2$ we modify slightly a result of Mostow [17] to show that there exists a constant $R>0$ such that for any $r$-flat $F^{*}$ in $H^{*}$ there exists an $r$-flat $F$ in $H$ with $\mathrm{Hd}\left(f\left(F^{*}\right), F\right)<R$. Here $r$ is the rank of $H^{*}$ and $\mathrm{Hd}($,$) denotes Hausdorff$ distance. In $\S 3$ we define and discuss the maximally singular geodesics of a symmetric space $H^{*}$ of noncompact type. We also provide a characterization of these geodesics in terms of a Cartan decomposition of the Lie algebra of $I_{0}\left(H^{*}\right)$. In $\S 4$ we use the results of $\S \S 2,3$ to show that a pseudoisometry $f: H^{*} \rightarrow H$ extends to a map $\bar{f}: S^{*} \rightarrow H(\infty)$, where $S^{*}$ denotes the set of maximally singular points in $H^{*}(\infty)$. One can show that the map $\bar{f}$ is one-one and continuous, but we do not need these properties and shall not prove them. In $\S 5$ we use the map $\bar{f}: S^{*} \rightarrow H(\infty)$ to a construct a family of closed, convex subsets $\left\{B_{p}: p \in H\right\}$, and we show that these sets are the leaves of a proper parallel foliation of $H$. In $\S 6$ we complete the proofs of theorems $A$ and $B$. The proofs of results in $\$ \S 4,5$ are quite long in several places and to make the exposition clearer we have put these proofs in Appendices 1 and 2.

\section{Preliminaries}

In this paper $H$ will always denote a complete, connected, simply connected Riemannian manifold of non-positive sectional curvature. $H$ will also be called a Hadamard manifold. For any two distinct points $p, q$ of $H$ there is a unique unit 
speed geodesic $\gamma_{p q}$ such that

$$
\gamma_{p q}(0)=p \text { and } \gamma_{p q}(a)=q, \quad \text { where } a=d(p, q) .
$$

All geodesics of $H$ will be assumed to have unit speed. We let $I(H)$ denote the group of isometries of $H$.

Points at infinity. (General reference [8].) If $\gamma, \sigma$ are any two geodesics of a Hadamard manifold $H$ we say that $\gamma$ and $\sigma$ are asymptotes if $d(\gamma t, \sigma t) \leq c$ for some positive constant $c$ and all $t \geq 0$. The equivalence class of asymptotic geodesics to which $\gamma$ belongs is denoted by $\gamma(\infty)$. By $\gamma(-\infty)$ we denote the equivalence class of the geodesic $\sigma(t)=\gamma(-t)$. We let $H(\infty)$ denote the set of all equivalence classes of geodesics of $H$. If $x$ and $y$ are distinct points of $H(\infty)$ we say that a geodesic $\gamma$ of $H$ joins $x$ and $y$ if either $\gamma(\infty)=x$ and $\gamma(-\infty)=y$ or $\gamma(\infty)=y$ and $\gamma(-\infty)=x$.

If $\bar{H}=H \cup H(\infty)$ then $\bar{H}$ admits a natural cone topology such that

(1) $\bar{H}$ is homeomorphic to the closed unit ball of dimension $n=\operatorname{dim} H$

(2) $H$ with its own topology is a dense open subset of $\bar{H}$.

Every isometry $\phi$ of $H$ extends to a homeomorphism of $\bar{H}$ by defining $\phi[\gamma(\infty)]=$ $(\phi \circ \gamma)(\infty)$.

If $p \in H$ and $x \in H(\infty)$ are given arbitrarily then there is a unique geodesic $\gamma_{p x}$ of $H$ such that $\gamma_{p x}(0)=p$ and $\gamma_{p x}(\infty)=x$. We define a continuous unit vector function

$$
V: H \times H(\infty) \rightarrow T_{1} H \quad \text { by } V(p, x)=\gamma_{p x}^{\prime}(0) .
$$

If $p, q, r$ are distinct points of $H$ we let $\chi_{p}(q, r)$ denote $\chi(V(p, q), V(p, r))$, the angle at $p$ subtended by $q$ and $r$. If $p \in H$ and $x, y \in H(\infty)$ are given arbitrarily we define

$$
\chi_{p}(x, y)=\Varangle(V(p, x), V(p, y)) .
$$

Angle measurement is continuous.

Limit sets. ([8]) If $\Gamma \subseteq I(H)$ is a group of isometries of $H$ we define $L(\Gamma)$, the limit set of $\Gamma$, to be the set $\overline{\Gamma(p)} \cap H(\infty)$. Here $\Gamma(p)$ denotes the orbit of a point $p$ in $H$ under $\Gamma$, and $\overline{\Gamma(p)}$ denotes the closure of $\Gamma(p)$ in $\bar{H}=H \cup H(\infty)$, relative to the cone topology. $L(\Gamma)$ is a closed subset of $H(\infty)$ that is invariant under $\Gamma$ and does not depend on the point $p$ in $H$. The set $L(\Gamma)$ is empty if and only if $\Gamma$ has a fixed point in $H$.

Busemann functions and horospheres. (General references $[3, \S 2]$ and $[8, \S 3]$.) If $\gamma$ is a geodesic of $H$ we define

$$
f=f_{\gamma}: H \rightarrow \mathbb{R} \quad \text { by } f(p)=\lim _{t \rightarrow+\infty} d(p, \gamma t)-t .
$$

If $\gamma$ belongs to a point $x \in H(\infty)$ we call $f$ a Busemann function at $x$. Any two Busemann functions at a given point $x$ in $H(\infty)$ differ by a constant. A Busemann function $f$ at $x$ in $H(\infty)$ is $C^{2}$ and convex. Moreover $(\operatorname{grad} f)(p) \doteq-V(p, x)$ for every point $p \in H([3],[8],[12])$. Given a point $x \in H(\infty)$, a point $p \in H$ and a Busemann function $f$ at $x$ we define

and

$$
L(p, x)=\{q \in H: f(q)=f(p)\}
$$

$$
B(p, x)=\{q \in H: f(q) \leq f(p)\} .
$$


$L(p, x)$ and $B(p, x)$ are called respectively the horosphere and horoball determined by $p$ and $x$.

Convexity. (General reference $[1, \S 1-\S 4]$.) A subset $A \subseteq H$ is convex if it contains the unique geodesic segment joining any two of its points. A continuous function $f: H \rightarrow \mathbb{R}$ is convex if the function $t \rightarrow(f \circ \gamma)(t)$ is a convex function on $\mathbb{R}$ for every geodesic $\gamma$ of $H$. If $f: H \rightarrow \mathbb{R}$ is $C^{2}$ then $f$ is convex if and only if the symmetric, bilinear form $\nabla^{2} f$ given by

$$
\nabla^{2} f(v, w)=\left\langle\nabla_{v} \operatorname{grad} f, w\right\rangle
$$

is positive semidefinite.

If $A \subseteq H$ is closed and convex then the function $p \rightarrow d(p, A)$ is a continuous convex function on $H$ and is differentiable if $A$ is a differentiable submanifold. For every point $p \in H$ there exists a unique point $P(p)$ in $A$ that is closest to $p$. The point $P(p)$ is called the foot of $p$ on $A$, and the unique geodesic from $p$ to $P(p)$ is called the perpendicular from $p$ to $A$.

The (orthogonal) projection map $P: H \rightarrow A$ has the property that $d(P p, P q) \leq$ $d(p, q)$ for all points $p, q$ in $H$. Moreover if

$$
d(P p, P q)=d(p, q)>0
$$

for points $p, q$ in $H-A$, then $p, q, P p$ and $P q$ are the vertices of a flat, totally geodesic, isometrically imbedded rectangle $R \subseteq H$; that is, there exists a totally geodesic, isometric imbedding $F: D \rightarrow H$, where $D \subseteq \mathbb{R}^{2}$ is a rectangle with the usual Euclidean metric [1, remark 3.3, p. 8].

Lattices and reducibility. ([5]) A Riemannian manifold $X$ is reducible if some finite Riemannian cover $\tilde{X}$ of $X$ can be expressed as a nontrivial Riemannian product $\tilde{X}=\tilde{X}_{1} \times \tilde{X}_{2}$. If $\boldsymbol{X}$ is a simply connected reducible manifold, then $\boldsymbol{X}$ itself is a nontrivial Riemannian product $X_{1} \times X_{2} . X$ is irreducible if it is not reducible.

If $\Gamma \subseteq I(H)$ is a freely acting, properly discontinuous group of isometries of a Hadamard manifold $H$, then $\Gamma$ is a lattice if the quotient manifold $H / \Gamma$ (hence also its unit tangent bundle) has finite Riemannian volume. A lattice $\Gamma$ is uniform (respectively non uniform) if $H / \Gamma$ is compact (respectively noncompact). A lattice $\Gamma$ is reducible (respectively irreducible) if the quotient manifold $H / \Gamma$ is reducible (respectively irreducible).

de Rham decompositions. (References [15] and [5].) It is evidently true that any Hadamard manifold $H$ can be written as a Riemannian product

$$
H=H_{0} \times H_{1} \times \cdots \times H_{k},
$$

where $H_{0}$ is a Euclidean space, possibly of dimension zero, and $H_{i}$ is an irreducible Hadamard manifold for $1 \leq i \leq k$. A theorem of de Rham states that this decomposition is unique modulo isometric equivalence and order of the factors. The manifolds $H_{i}, 0 \leq i \leq k$, are the de Rham factors of $H$. More generally the de Rham theorem applies to any Riemannian manifold $X$ and the Riemannian splitting is valid only locally if $X$ is not simply connected.

If $\phi$ is any isometry of $H$, then $\phi$ leaves invariant the foliation of $H$ corresponding to $H_{0}$ and permutes the foliations of $H$ corresponding to the non Euclidean de 
Rham factors $H_{i}, 1 \leq i \leq k$. We say that $\phi$ preserves the de Rham factors of $H$ if $\phi$ leaves invariant each of the foliations of $H$ corresponding to $H_{i}, 0 \leq i \leq k$. If $\phi$ preserves the de Rham factors of $H$, then $\phi$ has a unique expression

$$
\phi=\phi_{0} \times \phi_{1} \times \cdots \times \phi_{k}, \quad \text { where } \phi_{i} \in I\left(H_{i}\right) \text { for } 0 \leq i \leq k .
$$

If $\Gamma \subseteq I(H)$ is any subgroup then there exists a subgroup $\tilde{\Gamma} \subseteq \Gamma$ of finite index whose elements preserve the de Rham factors of $H$. For the group $\tilde{\Gamma}$ there are projection homomorphisms

determined by

$$
p_{i}: \tilde{\Gamma} \rightarrow I\left(H_{i}\right) \quad \text { for } 0 \leq i \leq k
$$

$$
p_{i}(\phi)=\phi_{i}, \quad \text { where } \phi=\phi_{0} \times \phi_{1} \times \cdots \times \phi_{k} .
$$

Symmetric spaces of noncompact type. (References [13], [14], [17].) A Hadamard manifold $H$ with no Euclidean de Rham factor $H_{0}$ is called a symmetric space (of noncompact type) if for every point $p \in H$ there exists an involutive isometry $S_{p}$ of $H$ such that $S_{p}$ fixes $p$ and

$$
S_{p}(\gamma t)=\gamma(-t)
$$

for every geodesic $\gamma$ with $\gamma(0)=p$. If $H$ is a symmetric space of noncompact type and if $G$ denotes $I_{0}(H)$, then $G$ is a transitive, noncompact, semisimple Lie group with trivial centre and no compact factors. The space $H$ may be identified with a coset space $G / K$ together with a $G$-invariant metric, where $K$ is the stability group of $G$ at some point $p$ in $H$. K is a maximal compact subgroup of $G$, and all such subgroups are stability groups, hence conjugate in $G$.

An $r$-flat in a Hadamard manifold $H$ is a totally geodesic, isometrically imbedded, closed submanifold of $H$ of dimension $r$ and sectional curvature zero. If $H$ is a symmetric space of noncompact type then its rank is the largest integer $r$ for which an $r$-flat exists in $H$. If $r$ is the rank of a symmetric space $H$, then every geodesic $\gamma$ of $H$ is contained in at least one $r$-flat. Moreover if $F_{1}, F_{2}$ are any two $r$-flats of $H$, then there is an isometry $\phi$ of $H$ such that $\phi\left(F_{1}\right)=F_{2}$.

Hausdorff distance. If $A \subseteq X$ is any subset of a complete Riemannian manifold $X$ and if $r^{*}$ is any positive number we define

$$
N_{r^{*}}(A)=\left\{x \in X: d(x, A) \leq r^{*}\right\} .
$$

If $A, B$ are any subsets of $X$ we define the Hausdorf distance between $A$ and $B$, denoted $\operatorname{Hd}(A, B)$ and possibly infinite, to be

Clearly

$$
\inf \left\{r^{*}>0: A \subseteq N_{r^{*}}(B) \text { and } B \subseteq N_{r^{*}}(A)\right\} \text {. }
$$

and

$$
\operatorname{Hd}(A, B)=\operatorname{Hd}(B, A)
$$

$$
\operatorname{Hd}(A, C) \leq \operatorname{Hd}(A, B)+\operatorname{Hd}(B, C)
$$

for any three subsets $A, B, C$ of $X$.

Pseudoisometries. (References [17] and [7].) If $M, M^{*}$ are any two homotopically equivalent manifolds, then they have isomorphic fundamental groups. Conversely if $M, M^{*}$ are both compact with non-positive sectional curvature, then any isomorphism of the fundamental groups induces a homotopy equivalence between $M$ and $M^{*}$. We explain briefly. 
Let $\Gamma, \Gamma^{*}$ denote uniform lattices in Hadamard manifolds $H, H^{*}$. Suppose that the quotient manifolds $H / \Gamma$ and $H^{*} / \Gamma^{*}$ have isomorphic fundamental groups, and let $\theta: \Gamma^{*} \rightarrow \Gamma$ be an explicit isomorphism. Following [17] or the slightly modified treatment in [7] one can construct a continuous map $f: H^{*} \rightarrow H$ with the following properties:

(1) $f$ is uniformly continuous;

(2) there exist positive constants $\xi^{*}, r^{*}$ such that

$$
\left(1 / \xi^{*}\right) d\left(p^{*}, q^{*}\right) \leq d\left(f p^{*}, f q^{*}\right) \leq \xi^{*} d\left(p^{*}, q^{*}\right)
$$

whenever $p^{*}, q^{*}$ are points in $H^{*}$ with $d\left(p^{*}, q^{*}\right) \geq r^{*}$;

(3) if $p^{*} \in H^{*}$ and $\phi^{*} \in \Gamma^{*}$ are given arbitrarily then

$$
f\left(\phi^{*} p^{*}\right)=\theta\left(\phi^{*}\right) f\left(p^{*}\right) \text {. }
$$

A map $f: H^{*} \rightarrow H$ satisfying the properties listed above will be called a $\theta$-equivariant $\left(\xi^{*}, r^{*}\right)$ pseudoisometry. Property (1) is given for emphasis only since it follows from (3). By the $\theta$-equivariance $f$ induces a continuous map $\tilde{f}$ between $H^{*} / \Gamma^{*}$ and $H / \Gamma$, and it is not difficult to show that $\tilde{f}$ is a homotopy equivalence.

We conclude this section with the following result that will be useful later:

Proposition 1. Let $\Gamma, \Gamma^{*}$ denote uniform lattices in Hadamard manifolds $H, H^{*}$, and let $\theta: \Gamma^{*} \rightarrow \Gamma$ be an isomorphism. Let $f: H^{*} \rightarrow H$ be a $\theta$-equivariant $\left(\xi^{*}, r^{*}\right)$ pseudoisometry, and let $\mathrm{g}: H \rightarrow H^{*}$ be a $\theta^{-1}$-equivariant $(\bar{\xi}, \bar{r})$ pseudoisometry, where $\bar{\xi}, \xi^{*}, \bar{r}$ and $r^{*}$ are appropriate positive constants. Then there exists a positive constant $\boldsymbol{A}$ such that:

(1) $d\left(p^{*}, g f p^{*}\right) \leq A$ for all $p^{*} \in H^{*}$

and (2) $d(p, f g p) \leq A$ for all $p \in H$.

Proof. Clearly it suffices to prove only (1). Suppose that (1) is false for every positive integer $n$ and choose a sequence $\left\{p_{n}^{*}\right\} \subseteq H^{*}$ such that $d\left(p_{n}^{*}, g f p_{n}^{*}\right) \geq n$ for every $n$. By the equivariance properties of $g$ and $f$ it follows that

$$
\phi^{*}\left(g f p^{*}\right)=g f\left(\phi^{*} p^{*}\right) \quad \text { for every } \phi^{*} \in \Gamma^{*} \text { and every } p^{*} \in H^{*} .
$$

Since $H^{*} / \Gamma^{*}$ is compact we may choose a sequence $\left\{\phi_{n}^{*}\right\} \subseteq \Gamma^{*}$ such that $\left\{q_{n}^{*}\right\}=$ $\left\{\phi_{n}^{*}\left(p_{n}^{*}\right)\right\}$ is a bounded sequence in $H$. Hence

$$
d\left(q_{n}^{*}, g f q_{n}^{*}\right)=d\left(p_{n}^{*}, g f p_{n}^{*}\right) \geqq n \quad \text { for every positive integer } n .
$$

By passing to a subsequence we may assume that $\left\{q_{n}^{*}\right\}$ converges to a point $q^{*} \in H^{*}$. It follows that

$$
d\left(q_{n}^{*}, g f q_{n}^{*}\right) \rightarrow d\left(q^{*}, g f q^{*}\right) \quad \text { as } n \rightarrow+\infty,
$$

contradicting the fact that $\left\{d\left(q_{n}^{*}, g f q_{n}^{*}\right)\right\}$ is unbounded by the choice of $p_{n}^{*}$ and $q_{n}^{*}$. Therefore assertion (1) is true, hence also (2).

\section{The association of r-flats}

This section is a modification of $[17, \S \S 13,14]$. Our main result is the following (compare [17, lemma 14.1]):

Proposition 2. Let $\Gamma^{*}$ be a uniform lattice in a symmetric space $H^{*}$ of noncompact type. Let $\Gamma$ be a uniform lattice in an arbitrary Hadamard manifold $H$, and let 
$\theta: \Gamma^{*} \rightarrow \Gamma$ be an isomorphism. Let $f: H^{*} \rightarrow H$ be a $\theta$-equivariant $\left(\xi^{*}, r^{*}\right)$ pseudoisometry for some positive constants $\xi^{*}, r^{*}$. Then there exists a positive constant $R$ with the following property:

if $r$ is the rank of $H^{*}$ then for any $r$-flat $F^{*}$ in $H^{*}$ there exists an $r$-flat $F$ in $H$ such that

$$
\operatorname{Hd}\left(f\left(F^{*}\right), F\right) \leq R
$$

We first need the following

LEMMA 2a. Let $g: H \rightarrow H^{*}$ be a $\theta^{-1}$-equivariant $(\bar{\xi}, \bar{r})$ pseudoisometry for some positive constants $\bar{\xi}, \bar{r}$. Let $G \subseteq \Gamma$ be a free abelian group of rank $r$, and let $G^{*}=\theta^{-1}(G) \subseteq \Gamma^{*}$. Let $F$ be an $r$-flat in $H$ such that $G$ leaves $F$ invariant and $F / G$ is compact. Let $F^{*}$ be the unique $r$-flat in $H^{*}$ such that $G^{*}$ leaves $F^{*}$ invariant and $F^{*} / G^{*}$ is compact. Then

$$
\operatorname{Hd}\left(g(F), F^{*}\right) \leq R^{*},
$$

where $R^{*}>0$ is a constant independent of the r-flats $F$ and $F^{*}$.

Remark. The existence of $r$-flats $F, F^{*}$ invariant under $G, G^{*}$ such that $F / G$ and $F^{*} / G^{*}$ are compact is a consequence of $[16$, theorem 1$]$. The uniqueness of $F^{*}$ is well known. See for example [17].

Proof. The proof of this result is contained in the proof of [17, lemma 13.2]. In that result it is assumed that both $H$ and $H$ are symmetric spaces of noncompact type, but the proof of lemma 13.2 is valid in our situation if only the target space $H^{*}$ is a symmetric space.

As a corollary we obtain

LEMMA 2b. There exists a positive constant $R$ with the following property: let $F^{*}$ be an $r$-flat in $H^{*}$ and $G^{*}$ a free abelian subgroup of $\Gamma^{*}$ of rank $r$ such that $G^{*}$ leaves $F^{*}$ invariant and $F^{*} / G^{*}$ is compact. Let $G=\theta\left(G^{*}\right) \subseteq \Gamma$ and let $F$ be any $r$-flat in $H$ such that $G$ leaves $F$ invariant and $F / G$ is compact. Then

$$
\operatorname{Hd}\left(f\left(F^{*}\right), F\right) \leq R \text {. }
$$

Proof. We first construct the constant $R$. Let $R^{*}>0$ be the constant of lemma 2 a. Since $g: H \rightarrow H^{*}$ is a $\theta^{-1}$-equivariant $(\bar{\xi}, \tilde{r})$ pseudosiometry it follows from the discussion of $\$ 1$ that for some positive constant $k$ we have

and

$$
d\left(p^{*}, g f p^{*}\right) \leq k \quad \text { for all } p^{*} \in H^{*}
$$

$$
d(p, f g p) \leq k \quad \text { for all } p \in H .
$$

Since $f$ is a $\left(\xi^{*}, r^{*}\right)$ pseudoisometry we have

$$
\left(1 / \xi^{*}\right) d\left(p^{*}, q^{*}\right) \leq d\left(f p^{*}, f q^{*}\right) \leq \xi^{*} d\left(p^{*}, q^{*}\right) \quad \text { if } d\left(p^{*}, q^{*}\right) \geq r^{*} .
$$

By the uniform continuity of $f$ we can choose a constant $\eta>0$ such that

$$
d\left(f p^{*}, f q^{*}\right) \leq \eta \text { whenever } d\left(p^{*}, q^{*}\right) \leq r^{*} \text {. }
$$

Let $\alpha=\max \left\{\xi^{*} R^{*}, \eta\right\}$ and let $R=k+\alpha$. 
We assert that $R$ has the desired properties. Let $F^{*}, F, G^{*}$ and $G$ be as in the statement of lemma 2 b. By lemma 2 a we have $\operatorname{Hd}\left(g(F), F^{*}\right) \leq R^{*}$. From the definition of $\alpha$ it follows that

$$
\operatorname{Hd}\left(f g(F), f\left(F^{*}\right) \leq \alpha .\right.
$$

Since $\mathrm{Hd}(f g(F), F) \leq k$ it follows that $\mathrm{Hd}\left(f\left(F^{*}\right), F\right) \leq k+\alpha=R$.

We now prove proposition 2. We assert that the constant $R$ of lemma $2 b$ also satisfies the condition of proposition 2. Let $F^{*}$ be an arbitrary $r$-flat of $H^{*}$. By [17, lemma 8.3] we can find a sequence $\left\{F_{n}^{*}\right\}$ of $r$-flats in $H^{*}$ and a sequence $\left\{G_{n}^{*}\right\}$ of free abelian subgroups of $\Gamma^{*}$ of rank $r$ such that $F_{n}^{*}$ converges to $F^{*}, G_{n}^{*}$ leaves $F_{n} / G_{n}$ is compact for every $n$. By lemma 2 b, $\operatorname{Hd}\left(f\left(F_{n}^{*}\right), F_{n}\right) \leq R$ for every $n$. Fix a not mean that $\mathrm{Hd}\left(F_{n}^{*}, F^{*}\right) \rightarrow 0$, which is false, but rather that every point $p^{*} \in F^{*}$ is a limit of a sequence $\left\{p_{n}^{*}\right\}$ with $p_{n}^{*} \in F_{n}^{*}$, and conversely if $\left\{p_{n}^{*}\right\}$ converges to $p^{*}$ with $p_{n}^{*} \in F_{n}^{*}$ for every $n$ then $p^{*} \in F^{*}$.

Let $G_{n}=\theta\left(G_{n}^{*}\right) \subseteq \Gamma$ and let $F_{n}$ be an $r$-flat such that $G_{n}$ leaves $F_{n}$ invariant and $F_{n} / G_{n}$ is compact for every $n$. By lemma $2 \mathrm{~b} \mathrm{Hd}\left(f\left(F_{n}^{*}\right), F_{n}\right) \leq R$ for every $n$. Fix a point $p^{*} \in F^{*}$ and let $\left\{p_{n}^{*}\right\}$ be a sequence converging to $p^{*}$ such that $p_{n}^{*} \in F_{n}^{*}$ for every $n$. Then $f\left(p_{n}^{*}\right) \rightarrow f\left(p^{*}\right)$ as $n \rightarrow \infty$ and it follows that for any number $\boldsymbol{R}^{\prime}>\boldsymbol{R}$ the $r$-flat $F_{n}$ intersects the closed ball of radius $R^{\prime}$ and centre $f\left(p^{*}\right)$ for all sufficiently large $n$. Hence some subsequence of the $r$-flats $\left\{F_{n}\right\}$ converges in the sense defined above to an $r$-flat $F$ in $H$. By continuity it follows that $\mathrm{Hd}\left(f\left(F^{*}\right), F\right) \leq R$ since

$$
F_{n}^{*} \rightarrow F^{*}, F_{n} \rightarrow F \quad \text { and } \operatorname{Hd}\left(f\left(F_{n}^{*}\right), F_{n}\right) \leq R \quad \text { for every } \mathrm{n} .
$$

This completes the proof of proposition 2 .

\section{Maximally singular geodesics and points at infinity}

Let $H^{*}$ denote a Riemannian symmetric space of noncompact type, and let $r$ denote the rank of $H^{*}$. As we noted in $\S 1$ every unit speed geodesic $\gamma^{*}$ of $H^{*}$ is contained in at least one $r$-flat. We say that a geodesic $\gamma^{*}$ of $H^{*}$ is singular if $\gamma^{*}(\mathbb{R})$ is contained in at least two distinct $r$-flats. A geodesic $\gamma^{*}$ is maximally singular if $\gamma^{*}(\mathbb{R})$ is the intersection of all $r$-flats of $H^{*}$ that contain $\gamma^{*}(\mathbb{R})$. If $r=1$ then every geodesic $\gamma^{*}$ is maximally singular. The maximally singular geodesics of $H^{*}$ correspond to the 1-dimensional simplices of the Tits complex associated to $H^{*}$ (cf. [17]), but we shall not pursue this view point. Maximally singular geodesics play an important part in the proof of theorem $\boldsymbol{A}$. In this section we discuss some properties of these geodesics including a proof of their existence and a characterization in terms of the Lie algebra of $I_{0}\left(H^{*}\right)$.

A point $x$ in $H^{*}(\infty)$ is said to be maximally singular if there exists a maximally singular geodesic $\gamma^{*}$ such that $\gamma^{*}(\infty)=x$. The next result will be useful later.

Proposition 3.1. Let $\gamma^{*}$ be a maximally singular geodesic of a symmetric space $H^{*}$ of noncompact type. If $\sigma$ is any geodesic asymptotic to $\gamma^{*}$, then $\sigma$ is also maximally singular. 
Proof. Let $x \in H^{*}(\infty)$ denote the asymptote class of geodesics to which $\gamma^{*}$ and $\sigma$ belong. Let $G$ denote $I_{0}\left(H^{*}\right)$ and let

$$
G_{x}=\{g \in G: g x=x\} .
$$

To prove the proposition it suffices to prove that $G_{x}$ is transitive on $H^{*}$, for then there exists an element $g \in G_{x}$ that carries $\gamma^{*}$ onto $\sigma$. The transitivity of $G_{x}$ is proved, for example, in [14, theorem 3.5.1]. We present here a short proof that is somewhat different. If $p, q$ are distinct points of $H^{*}$ we construct an element $\phi$ of $G_{x}$ such that $\phi p=q$. Choose $g \in G$ arbitrarily so that $g p=q$. Let $K$ be the subgroup of $G$ that fixes $p$. Then $K$ is a maximal compact subgroup of $G$ and by [2, prop. 4.4] there exists $k \in K$ such that $k x=g^{-1} x$. If we set $\phi=g k$, then $\phi x=x$ and $\phi p=q$.

Next we characterize maximally singular geodesics of $H^{*}$ in terms of the Lie algebra of $I_{0}\left(H^{*}\right)$. The characterization will also prove the existence of maximally singular geodesics of $H^{*}$.

We begin with a review of some basic facts about real semisimple Lie algebras. For further details see for example [13, pp. 121-125; 156-159; 173-174; 205; 214-219], [14, pp. 58-68] and [19, pp. 232-247]. See also [6, § 1]. We let $G, g$ denote the semisimple Lie group $I_{0}\left(H^{*}\right)$ and its Lie algebra. Let $B$ denote the Killing form on $g \times g$ given by

$$
B(X, Y)=\text { Trace }(\operatorname{ad} X \circ \text { ad } Y) \quad \text { for } X, Y \in g .
$$

$B$ is nondegenerate on $g \times g$ since $g$ is semisimple. Let $K$ be a maximal compact subgroup of $G$, and let $k$ denote the Lie algebra of $K$. Let $\mathscr{P}$ denote the orthogonal complement in $g$ of $k$ relative to $B$. We obtain a Cartan decomposition

$$
g=k+\mathscr{P}
$$

where $[k, k] \subseteq k,[k, \mathscr{P}] \subseteq \mathscr{P}$ and $[\mathscr{P}, \mathscr{P}] \subseteq \mathcal{k}$. Moreover $B$ is positive definite on $\mathscr{P} \times \mathscr{P}$ and negative definite on $k \times k$.

A Cartan subalgebra of $g$ is a maximal abelian subalgebra $h$ contained in $\mathscr{P}$ for some Cartan decomposition $=h+\mathscr{P}$. Any two Cartan subalgebras of $g$ are conjugate by an element of $\operatorname{Ad}(G) \subseteq \mathrm{GL}(g)$. The rank of $g$ is the dimension of a Cartan subalgebra of and is also equal to the rank of $H^{*}$ as we shall see.

Let $p^{*} \in H^{*}$ be fixed. We use the Cartan decomposition of that corresponds to $p^{*}$ to describe the geodesics of $H^{*}$ emanating from $p^{*}$ and the $r$-flats of $H^{*}$ that contain $p^{*}$, where $r$ is the rank of $H^{*}$ and $g$. If $K$ denotes the subgroup of $G$ that fixes $p^{*}$, then $K$ is a maximal compact subgroup of $G$. If $=\mathscr{k}+\mathscr{P}$ is the corresponding Cartan decomposition, then the geodesics of $H^{*}$ beginning at $p^{*}$ are those curves of the form $t \rightarrow \exp (t X)\left(p^{*}\right)$, where $X \in \mathscr{P}$. The $r$-flats of $H^{*}$ that contain $p^{*}$ are of the form $\exp (h)(p)$, where $h \subseteq \mathscr{P}$ is a Cartan subalgebra.

Now let $g=h+\mathscr{P}$ be a fixed Cartan decomposition. We define an element $X \in \mathscr{P}$ to be maximally singular if the 1-dimensional subspace of $g$ that is generated by $X$ is the intersection of all Cartan subalgebras in $\mathscr{P}$ that contain $X$. In view of the remarks of the preceding paragraph it is easy to see that $X \in \mathscr{P}$ is maximally singular if and only if $\gamma(t)=\exp (t X)\left(p^{*}\right)$ is a maximally singular geodesic of $H^{*}$. Here $p^{*}$ is the unique point of $H$ fixed by a maximal compact subgroup $K$ of $G$ with Lie algebra $k$. 
Proposition 3.2. Let $g=\ell+\mathscr{P}$ be a fixed Cartan decomposition of $g$. Then $X \in \mathscr{P}$ is maximally singular if and only if $Z(X)$ is a maximal subalgebra among the collection of subalgebras $\{Z(Y): Y \in \mathscr{P}\}$.

Here $Z(X)=\{W \in g:[X, W]=0\}$, the centralizer of $X$ in $g$. Note that the existence of maximally singular elements of $\mathscr{P}$ is an obvious consequence of the proposition.

To begin the proof of the proposition we note that if $h \subseteq \mathscr{P}$ is a Cartan subalgebra, then $\mathrm{ad}(\boldsymbol{h})$ is an abelian group of linear transformations of $q$. Moreover one can show that the elements of ad $(h)$ are all diagonalizable and hence we can write $g$ as a direct sum

$$
g=g^{0}+\sum_{\alpha} g^{\alpha}
$$

where $g^{0}=\{W \in g:$ ad $(H)(W)=[H, W]=0$ for all $H \in h\}$ and $\alpha: h \rightarrow \mathbb{R}$ is a nonzero linear transformation such that

$$
\left[H, X^{\alpha}\right]=\operatorname{ad}(H)\left(X^{\alpha}\right)=\alpha(H) X^{\alpha}
$$

for every $H \in h$ and every $X^{\alpha} \in g^{\alpha}$. The linear transformations $\alpha$ that arise in this manner are called the roots relative to $h$.

We need two preliminary results, the first of which is routine and not proved here. See also [14, p. 64].

LEMMA 3.2a. Let $h \subseteq \mathscr{P}$ be a Cartan subalgebra, and let $X \in \mathcal{h}$. Then

$$
Z(X)=g^{0}+\sum_{\alpha(X)=0} g^{\alpha}
$$

LEMMA 3.2b. Let $X \in \mathscr{P}$ be a given nonzero element, and let $\mathscr{P}_{X}$ denote the intersection of all Cartan subalgebras in $\mathscr{P}$ that contain $X$. If $Y \in \mathscr{P}$ is arbitrary then $Y \in \mathscr{P}_{X}$ if and only if $Z(Y) \supseteq Z(X)$.

Assuming for the moment that lemma $3.2 \mathrm{~b}$ has been proved we shall complete the proof of proposition 3.2. Suppose first that $X \in \mathscr{P}$ is maximally singular. Then by definition $\mathscr{P}_{X}$ is the 1-dimensional subspace of $\mathscr{P}$ generated by $X$. Let $Y \in \mathscr{P}$ be a non-zero element such that $Z(Y) \supseteq Z(X)$. Then $Y \in \mathscr{P}_{X}$ by lemma $3.2 \mathrm{~b}$ and hence $Y=t X$ for some $t \neq 0$. Therefore $Z(Y)=Z(X)$, which proves that $Z(X)$ is maximal among the set of subalgebras $\{Z(Y): Y \in \mathscr{P}\}$.

Next suppose that $X \in \mathscr{P}$ has the property that $Z(X)$ is maximal in $\{Z(Y): Y \in \mathscr{P}\}$. Let $Y \in \mathscr{P}_{X}$ be given arbitrarily. We wish to show that $Y=t X$ for some number $t$. By lemma 3.2b we see that $Z(Y) \supseteq Z(X)$, which implies that $Z(Y)=Z(X)$ by the maximality of $Z(X)$. Now let $h \subseteq \mathscr{P}$ be a Cartan subalgebra that contains $X$. Then $Y \in \mathscr{P}_{X} \subseteq h$. It follows from lemma 3.2a that for a root $\alpha$ of $h$ we have $\alpha(X)=0$ if and only if $\alpha(Y)=0$.

The Killing form $B$ is positive definite on $\mathscr{P} \times \mathscr{P}$ and hence on $h \times h$. For each non zero root $\alpha$ of $h$ we define $H^{\alpha} \in h$ by requiring that

$$
B\left(H^{\alpha}, H\right)=\alpha(H) \quad \text { for every } H \in h .
$$

For an element $W \in h$ we define

$$
h_{W}=\operatorname{span}\left\{H^{\alpha}: \alpha(W)=0\right\} .
$$


By the discussion of the preceding paragraph we see that $h_{Y}=h_{X}$. It suffices to show that $h_{X}=h_{Y}$ is a hyperplane in $h$, or equivalently that the orthogonal complement in $h$ relative to $B, h_{X}^{\perp}=h_{Y}^{\perp}$, is 1 -dimensional. If we know this then $X$ and $Y$ must be collinear since $X \in h_{X}^{\perp}$ and $Y \in h_{Y}^{\perp}$. This will show that $X$ is a maximally singular element of $\mathscr{P}$.

Suppose that $h_{X}=h_{Y}$ is not a hyperplane in $h$. Since

$$
h=\operatorname{span}\left\{H^{\alpha}: \alpha \text { is a non-zero root of } h\right\},
$$

$\left[17\right.$, p. 12], we can find a root $\beta$ of $h$ so that $\beta(X) \neq 0$ and $h^{*}=\operatorname{span}\left\{h_{X}, H^{\beta}\right\}$ is a proper subspace of $h$. Let $X^{*}$ be a non-zero element of $\left(h^{*}\right)^{\perp} \subseteq h$. Then $\beta\left(X^{*}\right)=0$ and $\alpha\left(X^{*}\right)=0$ for every root $\alpha$ such that $\alpha(X)=0$. By lemma 3.2a $Z\left(X^{*}\right)$ is strictly larger than $Z(X)$, contradicting the maximality of $Z(X)$. Therefore $h_{X}$ is a hyperplane in $h$ and $X$ is maximally singular.

We complete the proof of proposition 3.2 by proving lemma $3.2 b$. First let $X$, $Y$ be elements of $\mathscr{P}$ such that $Z(Y) \supseteq Z(X)$, and let $h \subseteq \mathscr{P}$ be a Cartan subalgebra that contains $X$. Since $h \subseteq Z(X)$ it follows that the vector subspace of $\mathscr{P}$ spanned by $h$ and $Y$ is an abelian subalgebra of $\mathscr{P}$. Since $h$ is a maximal abelian subalgebra it follows that $Y \in R$. Hence $Y \in \mathscr{P}_{X}$ since $h$ was arbitrary.

Next let $X \in \mathscr{P}$ be given, and let $Y \in \mathscr{P}_{X}$. Fix a Cartan subalgebra $h \subseteq \mathscr{P}$ that contains $X$. Since $\mathscr{P}_{X} \subseteq h$ it follows that $Y \in h$. To show that $Z(Y) \supseteq Z(X)$ it suffices by lemma 3.2a to show that $\alpha(Y)=0$ whenever $\alpha$ is a root of $h$ such that $\alpha(X)=0$.

Suppose that $\alpha(X)=0$ for some non-zero root $\alpha$ of $h$, and let $X^{\alpha}$ be a non-zero element of $g^{\alpha}$. We may write

$$
X^{\alpha}=K^{\alpha}+P^{\alpha}
$$

where $K^{\alpha} \in \mathcal{R}$ and $P^{\alpha} \in \mathscr{P}$. By hypothesis

$$
0=\alpha(X)\left(X^{\alpha}\right)=\left[X, X^{\alpha}\right]=\left[X, K^{\alpha}\right]+\left[X, P^{\alpha}\right]
$$

Since $X \in \mathscr{P},[\mathscr{P}, k] \subseteq \mathscr{P}$ and $[\mathscr{P}, \mathscr{P}] \subseteq \ell$ it follows that $\left[X, K^{\alpha}\right]=0$ and $\left[X, P^{\alpha}\right]=0$. Let $\tilde{h} \subseteq \mathscr{P}$ be a maximal abelian subalgebra that contains the abelian subalgebra generated by $X$ and $P^{\alpha}$. Then $Y \in \mathscr{P}_{X} \subseteq \tilde{h}$ and hence $\left[Y, P^{\alpha}\right]=0$. Finally

$$
\alpha(Y) K^{\alpha}+\alpha(Y) P^{\alpha}=\alpha(Y) X^{\alpha}=\left[Y, X^{\alpha}\right]=\left[Y, K^{\alpha}\right]+\left[Y, P^{\alpha}\right]=\left[Y, K^{\alpha}\right] .
$$

Since $[\mathscr{P}, \ell] \subseteq \mathscr{P}$ it follows that $\alpha(Y) K^{\alpha}=0$. If $K^{\alpha} \neq 0$ then $\alpha(Y)=0$ and we are done. If $K^{\alpha}=0$ then $P^{\alpha}=X^{\alpha} \neq 0$ and

$$
0=\left[Y, P^{\alpha}\right]=\left[Y, X^{\alpha}\right]=\alpha(Y) X^{\alpha}=\alpha(Y) P^{\alpha} .
$$

We see again that $\alpha(Y)=0$. Hence $\alpha(Y)=0$ whenever $\alpha(X)=0$, and it follows that $Z(Y) \supseteq Z(X)$ whenever $Y \in \mathscr{P}_{X}$. This completes the proof of lemma $3.2 \mathrm{~b}$.

4. Extension of a pseudoisometry $f: H^{*} \rightarrow H$ to the maximally singular points of $H^{*}(\infty)$

We consider the following situation: let $\Gamma^{*}$ denote a uniform lattice in a symmetric space $H^{*}$ of noncompact type and rank $r$; let $\Gamma$ denote a uniform lattice in an arbitrary Hadamard manifold $H$, and let $\theta: \Gamma^{*} \rightarrow \Gamma$ be an isomorphism; let $f: H^{*} \rightarrow H$ be a $\theta$-equivariant $\left(\xi^{*}, r^{*}\right)$ pseudoisometry for some positive constants $\xi^{*}, r^{*}$; let 
$S^{*} \subseteq H^{*}(\infty)$ denote the set of maximally singular points at infinity as defined in the previous section.

Our goal in this section is to define a $\theta$-equivariant map $\bar{f}: S^{*} \rightarrow H(\infty)$. In fact we can show that the map $\bar{f}$ is one-one and continuous but we shall not need these facts. Given a point $x^{*} \in S^{*}$ we let $\gamma^{*}$ be a geodesic representing $x^{*}$. It will follow from the result below that $x=\lim _{t \rightarrow+\infty}\left(f \circ \gamma^{*}\right)(t)$ exists in $H(\infty)$. We then define $\bar{f} x^{*}=x$. The value $x$ is independent of the geodesic $\gamma^{*}$ by the uniform continuity of $f$. Moreover it follows immediately from the $\theta$-equivariance of $f$ that

$$
\bar{f}\left(\phi^{*} x^{*}\right)=\theta\left(\phi^{*}\right)\left(\bar{f} x^{*}\right) \quad \text { for every } x^{*} \in S^{*} \text { and } \phi^{*} \in \Gamma^{*} .
$$

Proposition 4.1. Let $\Gamma^{*}, \Gamma, H^{*}, H, \theta$ and $f$ be as above. Let $\gamma^{*}$ be a maximally singular geodesic of $H^{*}$, and let $F^{*}$ be an r-flat containing $\gamma^{*}$. Let $F^{\prime}$ be an r-flat in $H$ such that $\mathrm{Hd}\left(f\left(F^{*}\right), F^{\prime}\right)<\infty$. Then

(1) the points $x=\lim _{t \rightarrow+\infty}\left(f \circ \gamma^{*}\right)(t)$ and $y=\lim _{t \rightarrow+\infty}\left(f \circ \gamma^{*}\right)(-t)$ exist and are distinct in $H(\infty)$;

(2) for any point $p$ in $F^{\prime}$ it follows that $\chi_{p}(x, y)=\pi$ and $\gamma_{p x}(\mathbb{R}) \subseteq F^{\prime}$.

Proof. Because of its length the proof of this result has been exiled to Appendix 1. We give here only a sketch that should convey the flavour of the argument. Since $\gamma^{*}$ is a maximally singular geodesic of $H^{*}$ we may choose $r$-flats $F_{1}^{*}, \ldots, F_{k}^{*}$ so that

$$
\gamma^{*}(\mathbb{R})=\bigcap_{i=1}^{k} F_{i}^{*}
$$

By proposition 2 we can choose a constant $R>0$ and $r$-flats $F_{1}, \ldots, F_{k}$ in $H$ so that

$$
\operatorname{Hd}\left(f\left(F_{i}^{*}\right), F_{i}\right)<R \quad \text { for every } i .
$$

Because $f$ is only a pseudoisometry and not an isometry we cannot conclude that the $r$-flats $F_{1}, \ldots, F_{k}$ have a nonempty intersection. However we can and do show that for some constant $R^{\prime}>0$ the tubular neighbourhoods $N_{R^{\prime}}\left(F_{1}\right), \ldots, N_{R^{\prime}}\left(F_{k}\right)$ of radius $R^{\prime}$ have a non-empty intersection that contains $\left(f \circ \gamma^{*}\right)(\mathbb{R})$. Because $\bigcap_{i=1}^{k} \mathrm{~F}_{i}^{*}$ is a single geodesic $\gamma^{*}$ which accumulates at $H^{*}(\infty)$ only at the two points $\gamma^{*}(\infty)$, $\gamma^{*}(-\infty)$ it is not surprising that $F=\bigcap_{i=1}^{k} N_{R^{\prime}}\left(F_{i}\right)$ accumulates at $H(\infty)$ only at two points $x, y$ and that these points can be joined by some geodesic $\sigma$ of $H$. (The technical difficulties in the proof occur here.) If $\sigma_{i}$ is the orthogonal projection of $\sigma$ onto $F_{i}$, then one can show that $\sigma_{i}$ is a geodesic of $H$ that joins $x$ to $y$ for each $i$. Finally $\chi_{p}(x, y)=\pi$ for every $i$ and every point $p \in F_{i}$ since each $F_{i}$ is an Euclidean space. This completes the outline of the proof.

We now consider the situation of theorem A where $H^{*}=H_{1}^{*} \times H_{2}^{*}$ is a reducible symmetric space of noncompact type. We regard $H_{1}^{*}(\infty)$ and $H_{2}^{*}(\infty)$ as subsets of $H^{*}(\infty)$. We let $S^{*} \subseteq H^{*}(\infty)$ denote the set of maximally singular points at infinity for $H^{*}$. For $i=1,2$ we define

$$
S_{i}^{*}=S^{*} \cap H_{i}^{*}(\infty)
$$

The sets $S_{1}^{*}, S_{2}^{*}$ are non-empty since any maximally singular point in $H_{i}^{*}(\infty)$ $(i=1,2)$ is easily seen to be a maximally singular point in $H^{*}(\infty)$. 
We let $\bar{f}: S^{*} \rightarrow H(\infty)$ be the $\theta$-equivariant map defined at the beginning of this section.

Definition 4.2. For $i=1,2$ let $S_{i}$ denote the closure in $H(\infty)$ of the set $\bar{f}\left(S_{i}^{*}\right)$.

One can actually show that $\bar{f}\left(S_{i}^{*}\right)$ is already closed in $H(\infty)$ for $i=1,2$, but we gain nothing by proving this.

Proposition 4.3. Let $\Gamma^{*}, \Gamma, H^{*}$ and $H$ be as defined at the beginning of this section. Then

(1) each of the sets $S_{1}, S_{2}$ is invariant under $\Gamma$;

(2) if $p \in H$ and $x \in S_{i}$ for $i=1,2$ are arbitrarily given points, then $\gamma_{p x}(-\infty) \in S_{i}$. In other words a point in $S_{i}$ can only be joined by a geodesic of $H$ to another point in $S_{i}$. Proof. (1) This follows immediately from the definition of $S_{i}$ and the fact that

$$
\bar{f}\left(\phi^{*} x^{*}\right)=\theta\left(\phi^{*}\right)\left(\bar{f} x^{*}\right) \quad \text { for every } x^{*} \in S_{i}^{*} \text { and every } \phi^{*} \in \Gamma^{*} \text {. }
$$

The maximally singular points $S^{*} \subseteq H^{*}(\infty)$ are invariant under $I\left(H^{*}\right)$.

(2) We consider only the case of $S_{1}$. We show first that if $p \in H$ and $x \in \bar{f}\left(S_{1}^{*}\right)$ are given arbitrarily then $\gamma_{p x}(-\infty) \in S_{1}$. Let $p \in H$ and $x=\bar{f}\left(x^{*}\right)$ be given, where $x^{*} \in S_{1}^{*}$. Let $\gamma^{*}$ be a geodesic of $H^{*}$ with $\gamma^{*}(\infty)=x^{*}$. By definition

$$
x=\bar{f}\left(x^{*}\right)=\lim _{t \rightarrow+\infty}\left(f \circ \gamma^{*}\right)(t) \text {. }
$$

By proposition $4.1 y=\lim _{t \rightarrow+\infty}\left(f \circ \gamma^{*}\right)(-t)$ also exists in $H(\infty)$, and $x$ can be joined to $y$ by a geodesic of $H$.

We assert that $y \in S_{1}$. Observe that $\gamma^{*}$ can be identified with a geodesic of $H_{1}^{*}$ since $\gamma^{*}(\infty) \in H_{1}^{*}(\infty)$ and hence

$$
\gamma^{*}(-\infty)=y^{*} \in H_{1}^{*}(\infty)
$$

If $\sigma(t)=\gamma^{*}(-t)$, then $\sigma$ is also a maximally singular geodesic of $H^{*}$ since $\sigma(\mathbb{R})=$ $\gamma^{*}(\mathbb{R})$. Therefore $y^{*} \in S_{1}^{*}$ and

$$
y=\lim _{t \rightarrow+\infty}\left(f \circ \gamma^{*}\right)(-t)=\lim _{t \rightarrow+\infty}(f \circ \sigma)(t) \in \bar{f}\left(S_{1}^{*}\right) \subseteq S_{1} .
$$

Since $\Gamma$ satisfies the duality condition (cf. [2, p. 78]) it follows from [2, lemma 2.4a] that every point $z$ of $H(\infty)$ to which $x$ can be joined by a geodesic of $H$ must lie in $\overline{\Gamma(y)}$. We note that $\overline{\Gamma(\mathrm{y})} \subseteq \mathrm{S}_{1}$ since $y \in S_{1}$ and $S_{1}$ is a closed set invariant under $\Gamma$. Therefore if $p \in H$ and $x \in \bar{f}\left(S_{1}^{*}\right)$ are given arbitrarily we conclude that

$$
\gamma_{p x}(-\infty) \in S_{1} .
$$

Now let $p \in H$ and $x \in S_{1}$ be given arbitrarily. Let $\left\{x_{n}\right\} \subseteq \tilde{f}\left(S_{1}^{*}\right)$ be a sequence that converges to $x$. Then $\gamma_{p x_{n}}(-\infty)$ converges to $\gamma_{p x}(-\infty)$. By the previous paragraph $\gamma_{p x_{n}}(-\infty)$ lies in $S_{1}$ for every $n$, and hence $\gamma_{p x}(-\infty) \in S_{1}$ since $S_{1}$ is closed. The proof of proposition 4.3 is complete.

\section{Constructing candidates for the leaves of a parallel foliation of $H$}

Let $S_{1} \subseteq H(\infty)$ and $S_{2} \subseteq H(\infty)$ be the subsets defined in the previous section. For each point $p \in H$ we let $B_{p}$ denote the smallest closed convex subset of $H$ that contains $\gamma_{p x}(\mathbb{R})$ for every $x \in S_{1}$. We show in this section that each set $B_{p}$ is a complete proper totally geodesic submanifold of $H$ (proposition 5.6). Moreover 
the manifolds $B_{p}$ are the leaves of a parallel foliation of $H$ that induces a non-trivial Riemannian product decomposition of $H$ (proposition 5.7). Proving that $H$ is reducible will then be sufficient to prove theorem $\mathrm{A}$. The arguments in this section are very similar to those used in the proof of theorem 2 of [10].

PROPOSITION 5.1. The set $B_{p}$ is a proper subset of $H$ for every point $p$ in $H$.

For the proof see Appendix 2.

Proposition 5.2. Let $p$ be an arbitrary point of $H$, and let $r$ be an arbitrary point of $B_{p}$. Then $B_{r} \subseteq B_{p}$.

Proof. Let $A_{r}$ denote the union of all geodesics $\gamma_{r x}(\mathbb{R})$ as $x$ ranges over $S_{1}$. It suffices to show that $A_{r} \subseteq B_{p}$ since $B_{r}$ is the smallest closed convex subset of $H$ that contains $A_{r}$. Let $x \in S_{1}$ be given, and let $\gamma_{n}$ be the geodesic segment from $r$ to $\gamma_{p x}(n)$ for every integer $n$. If $t \geq 0$ is fixed then $\gamma_{n}(t)$ lies between $r$ and $\gamma_{p x}(n)$ for all sufficiently large $n$, and hence $\gamma_{n}(t)$ lies in $B_{p}$ for large $n$. It follows that

since

$$
\gamma_{n}(t) \rightarrow \gamma_{r x}(t) \quad \text { as } n \rightarrow+\infty
$$

$$
\gamma_{p x}(n) \rightarrow x \quad \text { as } n \rightarrow+\infty \text {. }
$$

Therefore $\gamma_{r x}[0, \infty] \subseteq B_{p}$ since $B_{p}$ is closed. Since $\gamma_{r x}(-\infty) \in S_{1}$ a similar argument shows that $\gamma_{r x}(-\infty, 0] \subseteq B_{p}$. Therefore $A_{r} \subseteq B_{p}$, since $x \in S_{1}$ was arbitrary, and it follows that $B_{r} \subseteq B_{p}$.

Definition 5.3. We say that a set $B_{p}$ is minimally convex if $B_{r}=B_{p}$ for every $r \in B_{p}$. Proposition 5.4. There exists a point $p \in H$ such that $B_{p}$ is minimally convex.

For the proof of the result above see Appendix 2. Our next step is to show that $B_{p}$ is minimally convex for every point $p \in H$. Let

$$
\tilde{H} \cdot=\left\{p \in H: B_{p} \text { is minimally convex }\right\} .
$$

Proposition 5.5. $\tilde{H}=H$.

Proof. Our method is to prove that $\tilde{H}$ is nonempty, closed in $H$, convex and invariant under the deckgroup $\Gamma$ of $M=H / \Gamma$. It is then routine to show $\tilde{H}=H$ since $L(\Gamma)=H(\infty)$.

By proposition 5.4 the set $\tilde{H}$ is non-empty. We show next that $\tilde{H}$ is invariant under $\Gamma$. Let $\phi \in \Gamma$ and $p \in \tilde{H}$ be given. It is easy to see that $\phi\left(B_{p}\right)=B_{\phi p}$ since $\phi$ leaves $S_{1}$ invariant (by the work of the previous section). If $r \in B_{\phi p}$ then $\phi^{-1} r \in B_{p}$ and $B_{\phi^{-1}}=B_{p}$ since $p \in \tilde{H}$. Therefore

$$
B_{r}=\phi\left(B_{\phi^{-1} r}\right)=\phi\left(B_{p}\right)=B_{\phi p} .
$$

This proves that $\phi p \in \tilde{H}$ and hence $\tilde{H}$ is invariant under $\Gamma$.

We show that $\tilde{H}$ is closed in $H$. Let $\left\{p_{n}\right\} \subseteq \tilde{H}$ be a sequence converging to a point $p$ in $H$. Let $q \in B_{p}$ be given arbitrarily. To show that $B_{q}=B_{p}$ it suffices by proposition 5.2 to show that $p \in B_{q}$. By lemma 5.5a of Appendix 2 we know that

$$
d\left(q, B_{p_{n}}\right) \leq d\left(p, B_{p_{n}}\right) \leq d\left(p, p_{n}\right) \rightarrow 0 \quad \text { as } n \rightarrow+\infty .
$$

Hence for every integer $n$ there exists $q_{n} \in B_{p_{n}}$ such that $d\left(q, q_{n}\right) \rightarrow 0$ as $n \rightarrow+\infty$. 
Since $p_{n} \in \tilde{H}$ it follows that $p_{n} \in B_{q_{n}}$ for every $n$. Hence by lemma 5.5a of Appendix 2 we have

$$
d\left(p_{n}, B_{q}\right) \leq d\left(q_{n}, B_{q}\right) \leq d\left(q_{n}, q\right) \rightarrow 0 \quad \text { as } n \rightarrow+\infty .
$$

If we choose $p_{n}^{*} \in B_{q}$ so that $d\left(p_{n}, B_{q}\right)=d\left(p_{n}, p_{n}^{*}\right)$ then $p_{n}^{*} \rightarrow p$ since $p_{n} \rightarrow p$ as $n \rightarrow+\infty$ by hypothesis. Finally $p \in B_{q}$ since $p_{n}^{*} \in B_{q}$ for every $n$ and $B_{q}$ is closed.

The proof that $\tilde{H}$ is convex is rather long and we postpone it to Appendix 2 . Finally we conclude that $\tilde{H}=H$ since $L(\Gamma)=H(\infty)$ and $\tilde{H}$ is nonempty, closed, convex and invariant under $\Gamma$.

Proposition 5.6. For each point $p \in H$ the set $B_{p}$ is a complete, totally geodesic submanifold of $H$. (Compare with $[10, p .551])$

Proof. Let $p \in H$ be given. To show that $B_{p}$ is a totally geodesic submanifold it suffices to show that if $q_{1}, q_{2}$ are two distinct points of $B_{p}$ then $B_{p}$ contains the entire maximal geodesic through $q_{1}$ and $q_{2}$. The completeness of $B_{p}$ will then follow immediately since $B_{p}$ is closed in $H$.

Let $p \in H$ be given and let $q_{1}, q_{2}$ be any two distinct points of $B_{p}$. Let $\gamma$ be the unit speed geodesic of $H$ such that $\gamma(0)=q_{1}$ and $\gamma(c)=q_{2}$, where $c=d\left(q_{1}, q_{2}\right)$. Suppose that $\gamma\left(t_{0}\right) \notin B_{p}$ for some $t_{0}>c$ or some $t_{0}<0$. We consider only the case $t_{0}>c$, but the other case is similar. Let

$$
t^{*}=\sup \left\{t \geq c: \gamma\left(t^{*}\right) \in B_{p}\right\} \text {. }
$$

Then $t^{*}<t_{0}$ and $q^{*}=\gamma\left(t^{*}\right) \in B_{p}$ since $B_{p}$ is closed. Let $\bar{q}=\gamma\left(t_{0}\right)$ and let $r$ be the foot of $q_{1}$ on $B_{\bar{q}}$. By lemma 5.5b of Appendix 2 it follows that $q_{1}$ is the foot of $r$ on $B_{p}$.

Next we show that $r$ does not lie on the geodesic $\gamma$. If $r=\gamma(t)$ for some $t>t_{0}$ then $d\left(q_{1}, \bar{q}\right)<d\left(q_{1}, r\right)$, contradicting the definition of $r$. If $r=\gamma(t)$ for $t^{*}<t \leq t_{0}$ then $d\left(r, q_{2}\right)<d\left(r, q_{1}\right)$, contradicting the fact that $q_{1}$ is the foot of $r$ on $B_{p}$. If $r=\gamma(t)$ for $0 \leq t \leq t^{*}$ then $r \in B_{p}$ and $B_{\bar{q}}=B_{r}=B_{p}$ by proposition 5.5, contradicting the fact that $\bar{q} \notin B_{p}$. Finally if $r=\gamma(t)$ for some $t<0$ then $q_{1}=\gamma(0)$ lies between $r$ and $\bar{q}=\gamma\left(t_{0}\right)$ and hence $q_{1} \in B_{\bar{q}}$. By proposition 5.5 it follows that $B_{p}=B_{q_{1}}=B_{\bar{q}}$, contradicting the fact that $\bar{q} \notin B_{p}$. Therefore $r$ does not lie on $\gamma$.

Now consider the geodesic triangle in $H$ with vertices $q_{1}, \bar{q}$ and $r$. The angle at $r$ is at least $\pi / 2$, for if it were smaller than $\pi / 2$ there would be points on the geodesic segment $\gamma_{r q}$ closer to $q_{1}$ than $r$. Similarly since $q_{1}$ is the foot of $r$ on $B_{p}$ it follows that the angle of the triangle at $q_{1}$ is at least $\pi / 2$. Therefore the sum of the interior angles of the geodesic triangle is greater than $\pi$, which cannot happen in a complete, simply connected Riemannian manifold of non-positive sectional curvature $[13$, p. 73]. The contradiction shows that every point of $\gamma$ must lie in $B_{p}$, which completes the proof of proposition 5.6.

Proposition 5.7. $\mathrm{H}$ is reducible.

Proof. Let $p, q$ be any two points of $H$. By propositions 5.5 and 5.6 the sets $B_{p}, B_{q}$ are minimally convex and moreover are complete totally geodesic submanifolds. By lemma 5.5b of Appendix 2 the functions $\xi \rightarrow d\left(\xi, B_{q}\right)$ and $\eta \rightarrow d\left(\eta, B_{p}\right)$ are 
constant on $B_{p}$ and $B_{q}$ respectively. Hence $B_{p}(\infty)=B_{q}(\infty)$ regarded as subsets of $H(\infty)$. If $X=B_{p}(\infty)$ for some point $p \in H$ then $\phi(X)=X$ for every $\phi \in \Gamma$ since $\phi\left(B_{p}\right)=B_{\phi p}$. Now since $L(\Gamma)=H(\infty)$ it follows from [6, prop. 2.2] that $H$ is isometric to the Riemannian product of $B_{p}$ with another manifold. Therefore $H$ is reducible.

\section{Proofs of theorems $A$ and $B$}

For the statements of theorems $\mathrm{A}$ and $\mathrm{B}$ see the introduction. To prove theorem A it suffices by [6, prop. 4.6] to show that $H$ is reducible. By proposition $5.7 H$ is reducible, which completes the proof. We remark that the proof of proposition 4.6 of [6] involves showing that the manifold $H$ in that situation is a symmetric space and then applying the irreducible case of the Mostow rigidity theorem.

We now prove theorem B. Since $M$ and $M^{*}$ are homotopically equivalent they have the same dimension. We prove the theorem by induction on the dimension $n$. The result is obviously true for $n=2$. Suppose now that the theorem is true for all dimensions $\leq N$ and let $M, M^{*}$ have dimension $N+1$. Let $j, k$ denote the number of factors in the de Rham decomposition of $H, H^{*}$.

We represent $M$ and $M^{*}$ as quotient manifolds $H / \Gamma$ and $H^{*} / \Gamma^{*}$, where $\Gamma, \Gamma^{*}$ are uniform lattices in $H, H^{*}$. We shall use the induction hypothesis in the case that both $\Gamma$ and $\Gamma^{*}$ are reducible and shall use theorem $A$ in the other cases.

We consider first the case that $H$ or $H^{*}$ is irreducible; that is, $j=1$ or $k=1$. Let $H^{*}$ be irreducible. We begin by showing that both $\Gamma^{*}$ and $\Gamma$ are irreducible lattices. Since $\Gamma^{*}$ and $\Gamma$ are isomorphic it suffices to show that $\Gamma^{*}$ contains no finite index subgroup $G^{*}$ that is a non-trivial direct product $A^{*} \times B^{*}$. Suppose that such a subgroup $G^{*}$ does exist. We note that $G^{*}$ has a trivial centre; if the centre of $G^{*}$ were non-trivial then by $[5$, theorem 5.1$] \Gamma^{*}$ would contain Clifford translations and by [18, theorem 1] $H^{*}$ would admit a non-trivial Euclidean de Rham factor, contrary to our hypothesis. It now follows by [10, theorem 2] (see also [16, theorem 2]) that $H^{*} / G^{*}$ splits as a non-trivial Riemannian product. Therefore $H^{*}$ is reducible, contrary to our hypothesis.

We have shown that both $\Gamma$ and $\Gamma^{*}$ are irreducible if $H$ or $H^{*}$ is irreducible. Assuming that $H^{*}$ is irreducible we now show that $H$ is irreducible, which will prove theorem $\mathrm{B}$ in the case that $j=1$ or $k=1$. By the discussion in $\S 1$ we may choose a finite index subgroup $\tilde{\Gamma}$ of $\Gamma$ that preserves the de Rham factors of $H$. Then $\tilde{\Gamma}$ is irreducible since $\Gamma$ is irreducible. If $H$ were reducible then it would follow from [6, prop. 4.7] that $H$ is a symmetric space of noncompact type. By theorem $A, M$ and $M^{*}$ would be isometric up to a constant multiple of the metric, which would imply that $H^{*}$ is reducible, contrary to our hypothesis. Therefore $H$ is irreducible.

We consider next the case that both $H$ and $H^{*}$ are reducible. To begin we consider the case that one of the lattices $\Gamma$ or $\Gamma^{*}$ is irreducible. If $\Gamma$ is irreducible then by [6, prop. 4.7] $H$ is a symmetric space of noncompact type. By theorem A we see that $M$ and $M^{*}$, hence also $H$ and $H^{*}$, are isometric up to a constant multiple of the metric. Theorem $B$ is obviously true in this case. 
Finally we consider the case that both $\Gamma$ and $\Gamma^{*}$ are reducible lattices. Choose non-trivial subgroups $A, B$ of $\Gamma$ and $A^{*}, B^{*}$ of $\Gamma^{*}$ such that $A$ is isomorphic to $A^{*}, B$ is isomorphic to $B^{*}$ and the direct products $A^{*} \times B^{*}, A \times B$ both have finite index in $\Gamma^{*}, \Gamma$. As we showed earlier, the group $A \times B$ has trivial centre. Hence by the splitting theorem of $[\mathbf{1 0}]$ or [16] we have a non-trivial Riemannian product decomposition $H=H_{1} \times H_{2}$ such that $A \subseteq I\left(H_{1}\right), B \subseteq I\left(H_{2}\right)$ and $H /(A \times B)$ is isometric to $\left(H_{1} / A\right) \times\left(H_{2} / B\right)$. Similarly $H^{*}$ is a Riemannian product $H_{1}^{*} \times H_{2}^{*}$ such that $H^{*} /\left(A^{*} \times B^{*}\right)$ is isometric to $\left(H_{1}^{*} / A^{*}\right) \times\left(H_{2}^{*} / B^{*}\right)$. Applying the induction hypothesis to the manifolds $H_{1} / A, H_{1}^{*} / A^{*}$ and $H_{2} / B, H_{2}^{*} / B^{*}$ we conclude that the number and dimensions of the de Rham factors of $H_{i}$ equal those of $H_{i}^{*}$ for $i=1,2$. The proof of theorem $\mathrm{B}$ is now complete, since the set of de Rham factors of $H$ or $H^{*}$ is the union of the de Rham factors of $H_{1}$ and $H_{2}$ or $H_{1}^{*}$ and $H_{2}^{*}$ respectively.

\section{Appendix 1}

In this appendix we give a detailed proof of proposition 4.1. It will be convenient to break the proof into several lemmas which we state first and prove later. We use the notation found in the statement of proposition 4.1.

LEMMA 4.1a. Let $H^{*}$ be a symmetric space of noncompact type, and let $F_{1}^{*}, \ldots, F_{n}^{*}$ be flat, totally geodesic imbedded submanifolds of $H^{*}$ of dimensions $r_{1}, \ldots, r_{n}$. Let $F^{*}=\bigcap_{i=1}^{n} F_{i}^{*}$ be non-empty. Then for any number $R^{*}>0$ there exists a number $T=T\left(R^{*}, F_{1}^{*}, \ldots, F_{n}^{*}\right)>0$ with the following property: let $\left\{p_{1}, \ldots, p_{n}\right\}$ be points of $H$ such that

$$
p_{i} \in F_{i}^{*} \quad \text { for every } i
$$

and

$$
d\left(p_{i}, p_{j}\right) \leq R^{*} \quad \text { for all } 1 \leq i, j \leq n .
$$

Then

$$
d\left(p_{i}, F^{*}\right) \leq T \quad \text { for all } 1 \leq i \leq n .
$$

LEMMA 4.1b. Let $\gamma^{*}$ be a maximally singular geodesic of $H^{*}$, and let $F_{1}^{*}, \ldots, F_{n}^{*}$ be r-flats of $H^{*}$ such that $\gamma^{*}(\mathbb{R})=\bigcap_{i=1}^{n} F_{i}^{*}$, where $r$ is the rank of $H^{*}$. Let $R>0$ be the constant of proposition 2 , and for each $i$ choose an r-flat $F_{i}$ in $H$ so that

$$
\operatorname{Hd}\left(f\left(F_{i}^{*}\right), F_{i}\right) \leq R .
$$

Let $F=\bigcap_{i=1}^{n} N_{R}\left(F_{i}\right)$ where $N_{R}\left(F_{i}\right)=\left\{q \in H: d\left(q, F_{i}\right) \leq R\right\}$. Then

(1) $\left(f \circ \gamma^{*}\right)(\mathbb{R}) \subseteq F$;

(2) there exists a constant $A>0$ such that

$$
F \subseteq N_{A}\left(f \gamma^{*}(\mathbb{R})\right)=\left\{q \in H: d\left(q, f \gamma^{*}(\mathbb{R})\right) \leq A\right\} .
$$

If $S$ is any noncompact subset of a Hadamard manifold $H$ we define $S(\infty)$ to be $\bar{S} \cap H(\infty)$, where $\bar{S}$ denotes the closure of $S$ in $\bar{H}=H \cup H(\infty)$ relative to the cone topology of $\bar{H},[8, \S 2]$. Equivalently, a point $x \in H(\infty)$ lies in $S(\infty)$ if and only if there exists a sequence $\left\{p_{n}\right\} \subseteq S$ such that $p_{n} \rightarrow x$ as $n \rightarrow+\infty$. 
LeMMA 4.1c. Let $F, F_{1}, \ldots, F_{n}$ be as defined in lemma 4.1b. Then $F(\infty) \subseteq$ $\bigcap_{i=1}^{n} F_{i}(\infty)$. Moreover let $x, y$ be distinct points of $F(\infty) \subseteq H(\infty)$. Then $\chi_{p}(x, y)=\pi$ for every point $p \in\left(F_{1} \cup \cdots \cup F_{n}\right)$. In particular $F(\infty)$ contains at most two points.

One can also show that $F(\infty)=\bigcap_{i=1}^{n} F_{i}(\infty)$ but we shall not need this fact.

LEMMA 4.1d. Let $\gamma^{*}$ be a maximally singular geodesic of $H^{*}$. Then

$$
x=\lim _{t \rightarrow+\infty}\left(f \circ \gamma^{*}\right)(t) \text { and } y=\lim _{t \rightarrow+\infty}\left(f \circ \gamma^{*}\right)(-t)
$$

exist in $H(\infty)$ and are distinct.

Assuming for the moment that the lemmas above have been established we prove proposition 4.1. We first reduce to the case that $\operatorname{Hd}\left(f\left(F^{*}\right), F^{\prime}\right) \leq R$, where $R$ is the positive constant of proposition 2 . Let $\gamma^{*}$ be a maximally singular geodesic of $H^{*}$, and let $F^{*}$ be an $r$-flat containing $\gamma^{*}(\mathbb{R})$, where $r$ is the rank of $H^{*}$. By proposition 2 there exists an $r$-fiat $F^{\prime \prime}$ in $H$ such that $\operatorname{Hd}\left(f\left(F^{*}\right), F^{\prime \prime}\right) \leq R$. Now let $F^{\prime}$ be an $r$-flat in $H$ such that $\mathrm{Hd}\left(f\left(F^{*}\right), F^{\prime}\right)<\infty$. Then $\mathrm{Hd}\left(F^{\prime}, F^{\prime \prime}\right)<\infty$. From the work of $[6, \S 2]$ it follows that the functions $\xi \rightarrow d\left(\xi, F^{\prime}\right)$ and $\eta \rightarrow d\left(\eta, F^{\prime \prime}\right)$ are constant on $F^{\prime \prime}$ and $F^{\prime}$ respectively. Moreover if $P: H \rightarrow F^{\prime}$ denotes the orthogonal projection then $P$ is an isometry of $F^{\prime \prime}$ onto $F^{\prime}$. In addition if $\sigma$ is any maximal geodesic of $H$ contained in $F^{\prime \prime}$, then $P \circ \sigma$ is a maximal geodesic of $H$ contained in $F^{\prime}$ and $\sigma$, $P \circ \sigma$ bound a flat strip in $H$ if $F^{\prime}$ and $F^{\prime \prime}$ are distinct. Therefore it suffices to prove proposition 4.1, in particular part (2), in the case that $\mathrm{Hd}\left(f\left(F^{*}\right), F^{\prime}\right) \leq R$.

Part (1) of proposition 4.1 clearly follows from lemma $4.1 \mathrm{~d}$ so we proceed to part (2). It is easy to see from the discussion in $\S 3$ of maximally singular geodesics that one can find finitely many $r$-flats $F_{1}^{*}, \ldots, F_{n}^{*}$ in $H^{*}$ such that $\gamma^{*}(\mathbb{R})=\bigcap_{i=1}^{n} F_{i}^{*}$. Clearly we may assume that the given $r$-flat $F^{*}$ that contains $\gamma^{*}$ is one of the $r$-flats $F_{i}^{*}$ by setting $F^{*}=F_{n+1}^{*}$ if necessary. Hence $F^{\prime}$ is one of the flats $F_{1}, \ldots, F_{n}$ as defined in lemma 4.1b. By lemma $4.1 \mathrm{c}$ we see that $\chi_{p}(x, y)=\pi$ for every point $p \in F^{\prime}$, where $x=\lim _{t \rightarrow+\infty}\left(f \circ \gamma^{*}\right)(t)$ and $y=\lim _{t \rightarrow+\infty}\left(f \circ \gamma^{*}\right)(-t)$. In particular $y=$ $\gamma_{p x}(-\infty)$ for every $p \in F^{\prime}$. By lemmas $4.1 \mathrm{~b}$ and 4 .1c the points $x, y$ lie in $F(\infty) \subseteq F^{\prime}(\infty)$, where $F$ is defined in lemma $4.1 \mathrm{~b}$. It follows that $\gamma_{p x}(\mathbb{R}) \subseteq F^{\prime}$, which completes the proof of Proposition 4.1.

We now prove the lemmas $4.1 \mathrm{a}-4.1 \mathrm{~d}$.

Proof of lemma 4.1a. We proceed by induction on the integer $n$. Suppose for the moment that the case $n=2$ has been proved. Now consider $n>2$ and assume that the lemma is true for all integers $m \leq n-1$. Let $F_{1}^{*}, \ldots, F_{n}^{*}$ be flat, totally geodesic, complete imbedded submanifolds of $H^{*}$ of dimensions $r_{1}, \ldots, r_{n}$. Suppose that $F^{*}=\bigcap_{i=1} F_{1}^{*}$ is nonempty.

Let $R^{*}>0$ be given arbitrarily. We construct a number

$$
T=T\left(R^{*}, F_{1}^{*}, \ldots, F_{n}^{*}\right)>0
$$

having the properties asserted in the lemma. Let $G=F_{1}^{*} \cap F_{2}^{*}$. Applying the lemma for the case $n=2$ we may choose a positive number $A=A\left(R^{*}, F_{1}^{*}, F_{2}^{*}\right)$ such that if $p_{1}^{*}, p_{2}^{*}$ are points of $F_{1}^{*}, F_{2}^{*}$ with $d\left(p_{1}^{*}, p_{2}^{*}\right) \leq R^{*}$ then $d\left(p_{i}^{*}, G\right) \leq A$ for $i=1$, 2. Now apply the induction hypothesis to $G, F_{3}^{*}, \ldots, F_{n}^{*}$ and choose a positive number $B=B\left(R^{*}+A, G, F_{3}^{*}, \ldots, F_{n}^{*}\right)$ such that if $p^{*} \in G$ and $p_{i}^{*} \in F_{i}^{*}, 3 \leq i \leq n$, 
are arbitrarily chosen points whose pairwise distances are at most $R^{*}+A$, then

$$
d\left(p^{*}, F^{*}\right) \leq B \text { and } d\left(p_{i}^{*}, F^{*}\right) \leq B \quad \text { for } 3 \leq i \leq n .
$$

Finally let $T=A+B$.

We assert that $T$ has the properties stated in lemma $4.1 \mathrm{a}$. Let $p_{1}, \ldots, p_{n}$ be points of $H$ such that $p_{i} \in F_{i}^{*}$ for every $i$ and $d\left(p_{i}, p_{i}\right) \leq R^{*}$ for every $1 \leq i, j \leq n$. We show that $d\left(p_{i}, F^{*}\right) \leq T$ for every $i$. Since $d\left(p_{1}, p_{2}\right) \leq R^{*}$ it follows that $d\left(p_{i}, p_{i}^{\prime}\right) \leq A$ for $i=1,2$ and suitable points $p_{1}^{\prime}, p_{2}^{\prime}$ in $G=F_{1}^{*} \cap F_{2}^{*}$. Now consider the points $p_{1}^{\prime}, p_{3}, \ldots, p_{n}$. Clearly $F^{*}=G \cap \bigcap_{i=3}^{n} F_{i}^{*}$. Moreover

$$
d\left(p_{1}^{\prime}, p_{j}\right) \leq d\left(p_{1}^{\prime}, p_{1}\right)+d\left(p_{1}, p_{j}\right) \leq R^{*}+A \quad \text { for all } 3 \leq j \leq n .
$$

By the choice of $B$ we may conclude that

$$
d\left(p_{1}^{\prime}, F^{*}\right) \leq B \text { and } d\left(p_{j}, F^{*}\right) \leq B \quad \text { for all } 3 \leq j \leq n .
$$

Hence

$$
d\left(p_{1}, F^{*}\right) \leq d\left(p_{1}, p_{1}^{\prime}\right)+d\left(p_{1}^{\prime}, F^{*}\right) \leq A+B=T .
$$

Applying the same argument to the points $p_{2}^{\prime}, p_{3}, \ldots, p_{n}$ we conclude that $d\left(p_{2}, F^{*}\right) \leq T$. Since

$$
d\left(p_{i}, F^{*}\right) \leq B<T=A+B \quad \text { for all } 3 \leq j \leq n
$$

the proof of the lemma is complete.

It remains only to prove lemma $4.1 \mathrm{a}$ in the case $n=2$. Let $F_{1}^{*}, F_{2}^{*}$ be distinct complete, flat, totally geodesic imbedded submanifolds of $H^{*}$ of dimensions $r_{1}, r_{2}$. Suppose that $F^{*}=F_{1}^{*} \cap F_{2}^{*}$ is nonempty. For each point $q \in F^{*}$ let

$$
\theta(q)=\inf \left\{\chi\left(v_{1}, v_{2}\right): v_{i} \in T_{q}\left(F_{i}^{*}\right) \text { and } v_{i} \text { is orthogonal to } T_{q}\left(F^{*}\right) \text { for } i=1,2\right\} .
$$

We assert that $\theta \equiv \theta_{0}>0$ in $F^{*}$. We observe that $\theta(q)>0$ for every $q \in F^{*}$ since the submanifolds $F_{1}^{*}, F_{2}^{*}$ are distinct, totally geodesic and have $F^{*}$ as their intersection. We let $q, q^{*}$ be distinct points of $F^{*}$. Let

$$
v_{i} \in T_{q}\left(F_{i}^{*}\right) \cap T_{q}\left(F^{*}\right)^{\perp}
$$

be given for $i=1,2$ and let $v_{i}^{*} \in T_{q^{*}}\left(H^{*}\right)$ be obtained by parallel translation of $v_{i}$ along the geodesic segment from $q$ to $q^{*}$, which is contained in $F^{*}$. Then

$$
v_{i}^{*} \in T_{q^{*}}\left(F_{i}^{*}\right) \cap T_{q^{*}}\left(F^{*}\right)^{\perp} \quad \text { for } i=1,2
$$

since $F_{i}^{*}, F^{*}$ are totally geodesic. Hence $\Varangle\left(v_{1}, v_{2}\right)=\chi\left(v_{1}^{*}, v_{2}^{*}\right)$, and it follows that $v \equiv \theta_{0}>0$ in $F^{*}$.

Now let $R^{*}>0$ be given and define

$$
T=R^{*}+R^{*}\left[1+\left(2 / 1-\cos \theta_{0}\right)\right]^{\frac{1}{2}}
$$

We show that $T$ has the property asserted in the statement of lemma $4.1 \mathrm{a}$ when $n=2$.

Let $p_{1}, p_{2}$ be points of $H^{*}$ such that $p_{i} \in F_{i}^{*}$ for $i=1,2$ and $d\left(p_{1}, p_{2}\right) \leq R^{*}$. We show that $d\left(p_{i}, F^{*}\right) \leq T$ for $i=1,2$. Let $P: H^{*} \rightarrow F^{*}$ denote the orthogonal projection, and let $q_{i}=P\left(p_{i}\right)$ for $i=1,2$. Let

$$
t_{i}=d\left(p_{i}, q_{i}\right)=d\left(p_{i}, F^{*}\right) \quad \text { for } i=1,2 .
$$


We may assume that both $t_{1}$ and $t_{2}$ are positive for otherwise we are done immediately. For $i=1,2$ let

$$
v_{i} \in T_{q_{i}}\left(F^{*}\right)^{\perp} \cap T_{q_{i}}\left(F_{i}^{*}\right)
$$

be that unit vector such that $p_{i}=\exp _{q_{i}}\left(t_{i} v_{i}\right)$. Let $v_{2}^{*}$ be the vector obtained by parallel translation in $H^{*}$ of $v_{2}$ along the unique geodesic from $q_{2}$ to $q_{1}$. Note that

$$
v_{2}^{*} \in T_{q_{1}}\left(F_{2}^{*}\right) \cap T_{q_{1}}\left(F^{*}\right)^{\perp} \text {. }
$$

It follows that

$$
\alpha=\Varangle\left(v_{1}, v_{2}^{*}\right) \geq \theta_{0}>0
$$

by the definition of $\theta_{0}$ above.

Let $p_{2}^{*}=\exp _{q_{1}}\left(t_{2} v_{2}^{*}\right)$ and consider the geodesic triangle $\Delta$ in $H^{*}$ with vertices $q_{1}, p_{1}, p_{2}^{*}$ and vertex angle $\alpha$ at $q_{1}$. By definition we see that

$$
t_{2}=d\left(p_{2}, q_{2}\right)=d\left(p_{2}^{*}, q_{1}\right) \text {. }
$$

The Euclidean geometry of $F_{2}^{*}$ shows that

$$
d\left(p_{2}, p_{2}^{*}\right)=d\left(q_{1}, q_{2}\right)=d\left(P p_{1}, P p_{2}\right) \leq d\left(p_{1}, p_{2}\right) \leq R^{*}
$$

by [1, lemma 3.2]. If $c=d\left(p_{1}, p_{2}^{*}\right)$, the length of the side of $\Delta$ opposite $q_{1}$, then

$$
c \leq d\left(p_{1}, p_{2}\right)+d\left(p_{2}, p_{2}^{*}\right) \leq 2 R^{*} \text {. }
$$

Since $t_{1}=d\left(p_{1}, q_{1}\right)$ we may apply the law of cosines, as stated in $[8$, p. 47] or $[13$, p. 73], to $\Delta$ and conclude that

$$
\begin{aligned}
c^{2} \geq t_{1}^{2}+t_{2}^{2}-2 t_{1} t_{2} \cos \alpha & \geq t_{1}^{2}+t_{2}^{2}-2 t_{1} t_{2} \cos \theta_{0} \\
& =\left(t_{1}-t_{2}\right)^{2}+2 t_{1} t_{2}\left(1-\cos \theta_{0}\right) .
\end{aligned}
$$

From the inequalities above we see that

$$
c^{2} \geq \delta t_{1} t_{2}, \quad \text { where } \delta=2\left(1-\cos \theta_{0}\right)>0
$$

and

$$
\left|t_{1}-t_{2}\right| \leq c \leq 2 R^{*} \quad \text { since } c^{2} \geq\left(t_{1}-t_{2}\right)^{2}
$$

From these inequalities we see that $t_{2} \geq t_{1}-2 R^{*}$ and

$$
4\left(R^{*}\right)^{2} \geq c^{2} \geq \delta t_{1} t_{2} \geq \delta t_{1}\left(t_{1}-2 R^{*}\right) .
$$

Therefore

$$
\left(4\left(R^{*}\right)^{2} / \delta\right)+\left(R^{*}\right)^{2} \geq\left(t_{1}-R^{*}\right)^{2}
$$

and hence

$$
\left|t_{1}-R^{*}\right| \leq R^{*}[1+(4 / \delta)]^{\frac{1}{2}}=R^{*}[1+(2 / 1-\cos \theta)]^{\frac{1}{2}}
$$

We conclude that

$$
t_{1} \leq R^{*}+\left|t_{1}-R^{*}\right| \leq R^{*}+R^{*}[1+(2 / 1-\cos \theta)]^{\frac{1}{2}}=T
$$

This shows that

$$
d\left(p_{1}, F^{*}\right)=d\left(p_{1}, q_{1}\right)=t_{1} \leq T
$$

Similarly

$$
d\left(p_{2}, F^{*}\right)=d\left(p_{2}, q_{2}\right)=d\left(p_{2}^{*}, q_{1}\right)=t_{2} \leq T,
$$

which completes the proof of the lemma for the case $n=2$. 
Proof of lemma 4.1b. Assertion (1) is clear from the definition of $F$. We prove (2). We suppose that $F$ is not contained in $N_{A}\left(f \gamma^{*}(\mathbb{R})\right)$ for any positive number $A$ and derive a contradiction. Choose a sequence $\left\{p_{k}\right\} \subseteq F$ such that

$$
d\left(p_{k}, f \gamma^{*}(\mathbb{R})\right)=r_{k} \rightarrow+\infty \quad \text { as } k \rightarrow+\infty .
$$

Now fix an integer $k>0$. By the definition of $F$ we can find points $p_{k}^{i}, 1 \leq i \leq n$, such that $d\left(p_{k}, p_{k}^{i}\right) \leq R$ and $p_{k}^{i} \in F_{i}$ for every $i$.

Let $g: H \rightarrow H^{*}$ be a $\theta^{-1}$-equivariant pseudoisometry. By the discussion of $\S 1$ there exists a constant $M_{1}>0$ such that

(1) $d\left(p^{*}, g f p^{*}\right) \leq M_{1} \quad$ for all $p^{*} \in H^{*}$.

By the uniform continuity of $g$ there also exists a positive constant $M_{2}$ such that

(2) $d(g p, g q) \leq M_{2} \quad$ if $d(p, q) \leq 2 R$,

where $p, q$ are points of $H$, and $R$ is the constant of proposition 2. From (2) we obtain

(3) $d\left(g p_{k}, g p_{k}^{i}\right) \leq M_{2} \quad$ for $1 \leq i \leq n$.

Similarly since $d\left(p_{k}^{i}, p_{k}^{i}\right) \leq d\left(p_{k}^{i}, p_{k}\right)+d\left(p_{k}, p_{k}^{j}\right) \leq 2 R$ for $1 \leq i, j \leq n$ the inequality (2) also yields

(4) $d\left(g p_{k}^{i}, g p_{k}^{j}\right) \leq M_{2} \quad$ for $1 \leq i, j \leq n$.

Next, since $\operatorname{Hd}\left(f\left(F_{i}^{*}\right), F_{i}\right) \leq R$ for $1 \leq i \leq n$ we can find points $q_{k}^{i} \in F_{i}^{*}$ such that $d\left(f q_{k}^{i}, p_{k}^{i}\right) \leq R$ for all $1 \leq i \leq n$. From (2) we obtain

(5) $d\left(g f q_{k}^{i}, g p_{k}^{i}\right) \leq M_{2} \quad$ for $1 \leq i \leq n$.

For $1 \leq i, j \leq n$ we observe from (1), (4) and (5) that

$$
\begin{aligned}
d\left(q_{k}^{i}, q_{k}^{i}\right) & \leq d\left(q_{k}^{i}, g f q_{k}^{i}\right)+d\left(g f q_{k}^{i}, g p_{k}^{i}\right)+d\left(g p_{k}^{i}, g p_{k}^{j}\right)+d\left(g p_{k}^{j}, g f q_{k}^{j}\right)+d\left(g f q_{k}^{j}, q_{k}^{j}\right) \\
& \leq M_{1}+M_{2}+M_{2}+M_{2}+M_{1}=2 M_{1}+3 M_{2} .
\end{aligned}
$$

Hence we obtain

(6) $d\left(q_{k}^{i}, q_{k}^{j}\right) \leq 2 M_{1}+3 M_{2} \quad$ for all $1 \leq i, j \leq n$.

By hypothesis $\gamma^{*}(\mathbb{R})=\bigcap_{i=1}^{n} F_{i}^{*}$ and hence by lemma 4.1a we can find a number

$$
T=T\left(2 M_{1}+3 M_{2}, F_{1}^{*}, \ldots, F_{n}^{*}\right)>0
$$

such that $d\left(q_{k}^{i}, \gamma^{*}(\mathbb{R})\right) \leq T$ for all $1 \leq i \leq n$. This inequality holds for any choice of $k$ since the inequalities (1)-(6) do not depend on $k$. Therefore we obtain

(7) $d\left(q_{k}^{i}, \gamma^{*}(\mathbb{R})\right) \leq T \quad$ for all $k$ and all $1 \leq i \leq n$.

We conclude the proof of lemma $4.1 \mathrm{~b}$ by obtaining a contradiction to (7). Since $d\left(p_{k}, f \gamma^{*}(\boldsymbol{R})\right)=r_{k} \rightarrow+\infty$ it follows from the fact that $g: H \rightarrow H^{*}$ is a $(\bar{\xi}, \bar{r})$ pseudoisometry for some positive constants $\bar{\xi}, \bar{r}$ that

$$
d\left(g p_{k}, g f \gamma^{*} t\right) \geq(1 / \bar{\xi}) r_{k}
$$

for all $t \in \mathbb{R}$ and all integers $k$ so large that $r_{k}>\bar{r}$. Let $k_{0}>0$ be chosen so that the inequality above holds for all $k \geq k_{0}$. Since $d\left(g f \gamma^{*} t, \gamma^{*} t\right) \leq M_{1}$ for every $t$ by (1) we obtain

(8) $d\left(g p_{k}, \gamma^{*}(\mathbb{R})\right) \geq(1 / \bar{\xi}) r_{k}-M_{1} \quad$ for all $k \geq k_{0}$. 
For any $1 \leq i \leq n$ and any positive integer $k$ we see from (1), (3) and (5) that

$$
\begin{aligned}
d\left(g p_{k}, q_{k}^{i}\right) & \leq d\left(q_{k}^{i}, g f q_{k}^{i}\right)+d\left(g f q_{k}^{i}, g p_{k}^{i}\right)+d\left(g p_{k}^{i}, g p_{k}\right) \\
& \leq M_{1}+M_{2}+M_{2}=2 M_{2}+M_{1} .
\end{aligned}
$$

Hence for any $t \in \mathbb{R}$ and any integer $k \geq k_{0}$ we obtain

$$
d\left(q_{k}^{i}, \gamma^{*} t\right) \geq d\left(g p_{k}, \gamma^{*} t\right)-d\left(g p_{k}, q_{k}^{i}\right) \geq(1 / \bar{\xi}) r_{k}-\left(2 M_{1}+2 M_{2}\right)
$$

by (8) and the previous line. Thus we have

(9) $d\left(q_{k}^{i}, \gamma^{*}(\mathbb{R})\right) \geq(1 / \bar{\xi}) r_{k}-\left(2 M_{1}+2 M_{2}\right)$

for all $k \geq k_{0}$ and all $1 \leq i \leq n$.

Since $r_{k} \rightarrow+\infty$ we find that (9) contradicts (7) for large $k$. This contradiction shows that $F \subseteq N_{A}\left(f \gamma^{*}(\mathbb{R})\right)$ for some positive number $A$ and completes the proof of lemma 4.1b.

Proof of lemma 4.1c. Let an integer $i$ with $1 \leq i \leq n$ and a point $p \in F_{i}$ be fixed. Let $R^{\prime}=d(p, F)$. We show first that the geodesic ray $\gamma_{p z}[0, \infty)$ is contained in $N_{R^{\prime}}(F) \cap F_{i}$ for any point $z \in F(\infty)=\bar{F} \cap H(\infty)$, where $\bar{F}$ denotes the closure of $F$ in $\bar{H}=H \cup$ $H(\infty)$ relative to the cone topology $[8, \S 2]$. It then follows immediately that $F(\infty) \subseteq F_{i}(\infty)$.

Let $z \in F(\infty)$ be given, and let $\left\{p_{n}\right\} \subseteq F$ be a sequence converging to $z$. By the convexity of the function $g \rightarrow d(q, F)$ (see $\S 1$ ) it follows that for every point $q$ on the geodesic segment $\gamma_{p p_{n}}$ we have

$$
d(q, F) \leq \max \left\{d(p, F), d\left(p_{n}, F\right)\right\}=R^{\prime} .
$$

Therefore for any point $t \geq 0$ we have

$$
d\left(\gamma_{p z} t, F\right)=\lim _{n \rightarrow \infty} d\left(\gamma_{p p_{n}} t, F\right) \leq R^{\prime},
$$

or equivalently, $\gamma_{p z}[0, \infty) \subseteq N_{R^{\prime}}(F)$. We show that $\gamma_{p z}[0, \infty) \subseteq F_{i}$, which shows that $\gamma_{p z}[0, \infty) \subseteq N_{R^{\prime}}(F) \cap F_{i}$ and also that $z \in F_{i}(\infty)$. Since $\left\{p_{n}\right\} \subseteq F \subseteq N_{R}\left(F_{i}\right)$ we may choose a sequence $\left\{q_{n}\right\} \subseteq F_{i}$ so that $d\left(p_{n}, q_{n}\right) \leq R$ for every $n$. The geodesic segments $\gamma_{p q_{n}}$ are contained in $F_{i}$ since $F_{i}$ is convex, and hence $\gamma_{p z}[0, \infty) \subseteq F_{i}$ since $F_{i}$ is closed and $z=\lim _{n \rightarrow \infty} p_{n}=\lim _{n \rightarrow \infty} q_{n}$. In particular $z \in F_{i}(\infty)$.

Now let $x, y$ be any two distinct points of $F(\infty)$, and let $p$ be any point of $F_{i}$. We shall assume that $0<\chi_{p}(x, y)<\pi$ and obtain a contradiction. If $R^{\prime}=d(p, F)$ then it follows from the discussion above that the geodesic rays $\gamma_{p x}[0, \infty)$ and $\gamma_{p y}[0, \infty)$ are both contained in $N_{R^{\prime}}(F) \cap F_{i}$. The set $N_{R^{\prime}}(F) \cap F_{i}$ is clearly closed and is convex by the convexity of the function $q \rightarrow d(q, F)$. Let $\Delta_{i}$ denote the smallest closed convex subset of $H$ that contains both $\gamma_{p x}[0, \infty)$ and $\gamma_{p y}[0, \infty)$. Clearly $\Delta_{i} \subseteq N_{R^{\prime}}(F) \cap F_{i}$. If we regard $F_{i}$ as a Euclidean space of dimension $r \geq 2$ and let $\mathbb{R}^{2}$ denote the subspace of $F_{i}$ spanned by the unit vectors $V(p, x)$ and $V(p, y)$ (see $\S 1$ ), then $\Delta_{i}$ is an infinite triangular sector in $\mathbb{R}^{2}$ with vertex $p$, since $\mathbb{R}^{2}$ is a closed convex subset of $H$.

From the previous paragraph and lemma $4.1 \mathrm{~b}$ we obtain

(1) $\Delta_{i} \subseteq N_{R^{\prime}}(F) \subseteq N_{\left(R^{\prime}+A\right)}\left(f \gamma^{*} \mathbb{R}\right)$ 
for a suitable positive number $A$ that does not depend on $i$. Now let $B>R^{\prime}+A$ be given. We show that there exists $q \in \Delta_{i}$ such that $d\left(q, f \gamma^{*} \mathbb{R}\right) \geq B$. This will contradict condition (1) above and will allow us to conclude that $\chi_{p}(x, y)=\pi$ for all points $p \in F_{i}$ and every $1 \leq i \leq n$.

We assert that there exist positive constants $\beta_{1}$ and $\beta_{2}$ such that the Euclidean area in $\mathbb{R}^{2}$ of $\mathbb{R}^{2} \cap\left[N_{B}\left(f \gamma^{*}[-t, t]\right)\right]$ is at most $\beta_{1} t+\beta_{2}$ for all numbers $t \geq 0$. Assume for the moment that this assertion has been proved. By hypothesis $f: H^{*} \rightarrow H$ is a $\left(\xi^{*}, r^{*}\right)$ pseudoisometry for some positive constants $\xi^{*}, r^{*}$ and it follows that

$$
\left(1 / \xi^{*}\right)|t-s| \leq d\left(f \gamma^{*} t, f \gamma^{*} s\right) \leq \xi^{*}|t-s| \quad \text { whenever }|t-s| \geq r^{*}
$$

Now fix a positive number $t$ so large that

$$
r^{*}<t^{*}=\xi^{*}\left[t+B+d\left(p, f \gamma^{*} 0\right)\right] .
$$

Let $\delta_{1}$ and $\delta_{2}$ be those positive constants such that

$$
\beta_{1} t^{*}+\beta_{2}=\delta_{1} t+\delta_{2}
$$

for every number $t$. Now choose $t$ even larger if necessary so that

$$
\alpha t^{2} / 2>\delta_{1} t+\delta_{2}
$$

where $0<\alpha=\chi_{p}(x, y)<\pi$.

We assert that there exists $q \in \Delta_{i}$ such that $d(p, q) \leq t$ and $d\left(q, f \gamma^{*} \mathbb{R}\right) \geq B$. As we explained above this will contradict condition (1). For each positive number $s$ let

$$
\Delta_{i}(s)=\left\{q \in \Delta_{i}: d(p, q) \leq s\right\} .
$$

The set $\Delta_{i}(s) \subseteq \mathbb{R}^{2}$ is a circular sector of radius $s$ and vertex $p$ whose Euclidean area in $\mathbb{R}^{2}$ equals $\alpha s^{2} / 2$. It follows that if $t$ is chosen as in the paragraph above then $\Delta_{i}(t)$ is not contained in $\mathbb{R}^{2} \cap\left[N_{B}\left(f \gamma^{*}\left[-t^{*}, t^{*}\right]\right)\right]$ since

$$
\text { area } \begin{aligned}
\Delta_{i}(t)=\alpha t^{2} / 2 & >\delta_{1} t+\delta_{2}=\beta_{1} t^{*}+\beta_{2} \\
& \geq \operatorname{area} \mathbb{R}^{2} \cap\left[N_{B}\left(f \gamma^{*}\left[-t^{*}, t^{*}\right]\right)\right] .
\end{aligned}
$$

We may therefore choose a point

$$
q \in \Delta_{i}(t)-\mathbb{R}^{2} \cap\left[N_{B}\left(f \gamma^{*}\left[-t^{*}, t^{*}\right]\right)\right] .
$$

By the choice of $q$ it follows that if

$$
|s| \leq t^{*}=\xi^{*}\left[t+B+d\left(p, f \gamma^{*} 0\right)\right]
$$

then

$$
d\left(q, f \gamma^{*} s\right) \geq d\left(q, f \gamma^{*}\left[-t^{*}, t^{*}\right]\right)>B .
$$

On the other hand, if $|s|>t^{*}$ then

$$
\begin{aligned}
d\left(q, f \gamma^{*} s\right) & \geq d\left(f \gamma^{*} 0, f \gamma^{*} s\right)-d(p, q)-d\left(p, f \gamma^{*} 0\right) \\
& \geq\left(1 / \xi^{*}\right)|s|-t-d\left(p, f \gamma^{*} 0\right) \\
& \geq\left(1 / \xi^{*}\right) t^{*}-t-d\left(p, f \gamma^{*} 0\right)=B .
\end{aligned}
$$

Hence $d\left(q, f \gamma^{*} \mathbb{R}\right) \geq B>R^{\prime}+A$, which contradicts condition (1).

It only remains to show that there exist positive constants $\beta_{1}, \beta_{2}$ such that area $\mathbb{R}^{2} \cap\left[N_{B}\left(f \gamma^{*}[-t, t]\right)\right]$ is at most $\beta_{1} t+\beta_{2}$ for all positive numbers $t$. By the uniform continuity of $f: H^{*} \rightarrow H$ we can choose a positive constant $M_{1}$ such that $d(f p, f q) \leq$ $M_{1}$ whenever $d(p, q) \leq 1$. For every integer $k$ we let $p_{k}$ denote $f \gamma^{*}(k)$. 
Let a positive number $t$ be given and let $m$ be the largest integer $\leq t$. Let $D_{k}$ denote the closed ball in $H$ with centre $p_{k}$ and radius $M_{1}+B$. We show that

$$
N_{B}\left(f \gamma^{*}[-t, t]\right) \subseteq \bigcup_{k=-m}^{m} D_{k}
$$

First let $s \in[-m, m]$ be given, and choose an integer $k \in[-m, m]$ so that $|k-s| \leq 1$. It follows that

$$
d\left(f \gamma^{*} s, p_{k}\right)=d\left(f \gamma^{*} s, f \gamma^{*} k\right) \leq M_{1} .
$$

Similarly if $m \leq s \leq t$ then $d\left(f \gamma^{*} s, p_{m}\right) \leq M_{1}$ and $d\left(f \gamma^{*}(-s), p_{-m}\right) \leq M_{1}$. Hence

$$
N_{B}\left(f \gamma^{*}[-t, t]\right) \subseteq \bigcup_{k=-m}^{m} D_{k}
$$

It follows that

$$
\mathbb{R}^{2} \cap\left[N_{B}\left(f \gamma^{*}[-t, t]\right)\right] \subseteq \bigcup_{k=-m}^{m}\left(D_{k} \cap \mathbb{R}^{2}\right) .
$$

If $D_{k} \cap \mathbb{R}^{2}$ is non-empty we choose a point $a_{k} \in D_{k} \cap \mathbb{R}^{2}$ and define

$$
D_{k}^{*}=\left\{q \in \mathbb{R}^{2}: d\left(q, a_{k}\right) \leq 2\left(M_{1}+B\right)\right\} .
$$

Clearly $D_{k} \cap \mathbb{R}^{2} \subseteq D_{k}^{*}$ and hence

$$
\text { area }\left(D_{k} \cap \mathbb{R}^{2}\right) \leq \text { area }\left(D_{k}^{*}\right)=4 \pi\left(M_{1}+B\right)^{2} .
$$

We conclude that

$$
\text { area } \begin{aligned}
\mathbb{R}^{2} \cap\left[N_{B}\left(f \gamma^{*}[-t, t]\right)\right] & \leq \sum_{k=-m}^{m} \text { area }\left(D_{k} \cap \mathbb{R}^{2}\right) \leq(2 m+1) 4 \pi\left(M_{1}+B\right)^{2} \\
& \leq(2 t+1) 4 \pi\left(M_{1}+B\right)^{2}=\beta_{1} t+\beta_{2},
\end{aligned}
$$

where $\beta_{1}=8 \pi\left(M_{1}+B\right)^{2}$ and $\beta_{2}=4 \pi\left(M_{1}+B\right)^{2}$. The proof of lemma $4.1 \mathrm{c}$ is now complete.

Proof of lemma 4.1d. Let $\gamma^{*}$ be a maximally singular geodesic of $H^{*}$. Clearly the geodesic $\sigma^{*}(t)=\gamma^{*}(-t)$ is also maximally singular. We show first that $x=$ $\lim _{t \rightarrow+\infty}\left(f \circ \gamma^{*}\right)(t)$ exists, and from this it follows that

$$
y=\lim _{t \rightarrow \infty}\left(f \circ \gamma^{*}\right)(-t)=\lim _{t \rightarrow+\infty}\left(f \circ \sigma^{*}\right)(t)
$$

also exists. Finally we show that $x \neq y$.

Clearly the curve $t \rightarrow\left(f \circ \gamma^{*}\right)(t)$ ultimately leaves any compact subset of $H$ as $t \rightarrow+\infty$ since $f$ is a pseudoisometry. Hence if $\left\{t_{n}\right\} \subseteq \mathbb{R}$ is any sequence such that $t_{n} \rightarrow+\infty$ as $n \rightarrow+\infty$ then there is a subsequence $\left\{t_{n_{k}}\right\}$ such that $\left(f \circ \gamma^{*}\right)\left(t_{n_{k}}\right)$ converges to some point of $H(\infty)$ as $k \rightarrow+\infty$. Suppose now that $\lim _{t \rightarrow+\infty}\left(f \circ \gamma^{*}\right)(t)$ does not exist. Then we can find distinct sequences $\left\{s_{n}\right\} \rightarrow+\infty$ and $\left\{t_{n}\right\} \rightarrow+\infty$ such that

$$
\left(f \circ \gamma^{*}\right)\left(s_{n}\right) \rightarrow z_{1} \in H(\infty)
$$

and

$$
\left(f \circ \gamma^{*}\right)\left(t_{n}\right) \rightarrow z_{2} \neq z_{1} \in H(\infty) \quad \text { as } n \rightarrow+\infty .
$$

It suffices to construct a sequence $\left\{r_{n}\right\} \rightarrow+\infty$ such that

$$
\left(f \circ \gamma^{*}\right)\left(r_{n}\right) \rightarrow z_{3} \in H(\infty),
$$


a point distinct from $z_{1}$ and $z_{2}$, for then $F(\infty)$ will contain the three points $z_{1}, z_{2}$ and $z_{3}$, contradicting lemma $4.1 \mathrm{c}$. We recall that $\left(f \circ \gamma^{*}\right)(\mathbb{R}) \subseteq F$ by lemma $4.1 \mathrm{~b}$, and hence $z_{i} \in F(\infty)$ for $1 \leq i \leq 3$.

By passing to a subsequence if necessary we may assume that $s_{n} \leq t_{n}$ for every $n$. It follows that $s_{n}<t_{n}$ for large $n$ since $z_{1} \neq z_{2}$. By continuity we may choose $r_{n} \in\left(s_{n}, t_{n}\right)$ so that

$$
\chi_{p}\left(f \gamma^{*} s_{n}, f \gamma^{*} r_{n}\right)=\chi_{p}\left(f \gamma^{*} r_{n}, f \gamma^{*} t_{n}\right),
$$

where $p$ is a fixed point in $H$. Let $\alpha_{n}$ denote this common angle. Since $z_{1} \neq z_{2}$ there exists $\varepsilon>0$ such that

$$
\Varangle_{p}\left(f \gamma^{*} s_{n}, f \gamma^{*} t_{n}\right) \geq \varepsilon>0 \quad \text { for large } n .
$$

Hence $\alpha_{n} \geq \varepsilon / 2$ for large $n$ since

$$
\chi_{p}\left(f \gamma^{*} s_{n}, f \gamma^{*} t_{n}\right) \leq \chi_{p}\left(f \gamma^{*} s_{n}, f \gamma^{*} r_{n}\right)+\chi_{p}\left(f \gamma^{*} r_{n}, f \gamma^{*} t_{n}\right)=2 \alpha_{n} .
$$

By passing to a subsequence $f \gamma^{*} r_{n} \rightarrow z_{3} \in F(\infty)$ and

$$
\chi_{p}\left(z_{3}, z_{1}\right)=\chi_{p}\left(z_{3}, z_{2}\right)=\lim _{n \rightarrow \infty} \alpha_{n} \geq \varepsilon / 2 .
$$

Therefore $z_{1}, z_{2}, z_{3}$ are distinct points of $F(\infty)$, which contradicts lemma $4.1 \mathrm{c}$. It follows that $x=\lim _{t \rightarrow+\infty}\left(f \circ \gamma^{*}\right)(t)$ exists in $H(\infty)$.

To complete the proof of lemma $4.1 \mathrm{~d}$ it only remains to show that $x, y$ are distinct points of $F(\infty)$. Suppose that $x=y$. Let $P_{1}: H \rightarrow F_{1}$ be the orthogonal projection, and let $\sigma_{1}(t)=P_{1}\left(f \gamma^{*} t\right)$. We show first that there exist positive constants $c_{1}, c_{2}$ and $r_{1}$ such that

$$
c_{1}|t-s| \leq d\left(\sigma_{1} t, \sigma_{1} s\right) \leq c_{2}|t-s|
$$

if $|t-s| \geq r_{1}$. By hypothesis $f: H^{*} \rightarrow H$ is a $\left(\xi^{*}, r^{*}\right)$ pseudoisometry which means that

$$
\left(1 / \xi^{*}\right)|t-s| \leq d\left(f \gamma^{*} t, f \gamma^{*} s\right) \leq \xi^{*}|t-s| \quad \text { whenever }|t-s| \geq r^{*} .
$$

Also by hypothesis we have $\operatorname{Hd}\left(f\left(F_{1}^{*}\right), F_{1}\right) \leq R$ which means that

$$
\text { - } d\left(\sigma_{1} t, f \gamma^{*} t\right)=d\left(f \gamma^{*} t, F_{1}\right) \leq R \quad \text { for every } t .
$$

The existence of the constants $c_{1}, c_{2}$ and $r_{1}$ that satisfy the inequalities above is now clear.

Let $q=\sigma_{1}(0)$ and choose sequences $\left\{t_{n}\right\} \rightarrow+\infty$ and $\left\{s_{n}\right\} \rightarrow+\infty$ such that

$$
d\left(q, \sigma_{1}\left(t_{n}\right)\right)=d\left(q, \sigma_{1}\left(-s_{n}\right)\right)=n
$$

for every positive integer $n$. Note that since $d\left(\sigma_{1} t, f \gamma^{*} t\right) \leq R$ for every $t$ we have

$$
x=\lim _{n \rightarrow \infty}\left(f \circ \gamma^{*}\right)\left(t_{n}\right)=\lim _{n \rightarrow \infty} \sigma_{1}\left(t_{n}\right)
$$

and

$$
y=\lim _{n \rightarrow \infty}\left(f \circ \gamma^{*}\right)\left(-s_{n}\right)=\lim _{n \rightarrow \infty} \sigma_{1}\left(-s_{n}\right) .
$$

If $\theta_{n}=\chi_{q}\left(\sigma_{1}\left(t_{n}\right), \sigma_{1}\left(-s_{n}\right)\right)$, then $\theta_{n} \rightarrow \chi_{q}(x, y)=0$ as $n \rightarrow+\infty$. If $d_{n}=d\left(\sigma_{1}\left(t_{n}\right), \sigma_{1}\left(-s_{n}\right)\right)$ then by the usual Euclidean law of cosines in $F_{1}$ we obtain $d_{n}^{2}=2 n^{2}\left(1-\cos \theta_{n}\right)$ or

$$
d_{n} / n=\left[2\left(1-\cos \theta_{n}\right)\right]^{\frac{1}{2}} \rightarrow 0 \quad \text { as } n \rightarrow+\infty .
$$


On the other hand we know that for large $n$ we obtain $n=d\left(\sigma_{1}(0), \sigma_{1}\left(-s_{n}\right)\right) \leq c_{2} s_{n}$ and $n=d\left(\sigma_{1}(0), \sigma_{1}\left(t_{n}\right)\right) \leq c_{2} t_{n}$. Hence for large $n$ we obtain the inequality

$$
\begin{aligned}
d_{n} & =d\left(\sigma_{1}\left(t_{n}\right), \sigma_{1}\left(-s_{n}\right)\right) \geq c_{1}\left(t_{n}+s_{n}\right) \\
& \geq c_{1}\left[\left(n / c_{2}\right)+\left(n / c_{2}\right)\right]=n\left(2 c_{1} / c_{2}\right) .
\end{aligned}
$$

Therefore $d_{n} / n \geq 2 c_{1} / c_{2}>0$ for large $n$, which contradicts the conclusion of the previous paragraph. This shows that $x \neq y$ and completes the proof of lemma 4.1d.

\section{Appendix 2}

In this section we give the proofs of various results in $\S 5$.

Proof of proposition 5.1. We begin with the following:

LEMMA 5.1. Let $H$ be an arbitrary Hadamard manifold, and let $x$, $y$ be arbitrary distinct points of $H(\infty)$. Let $C_{x y}$ denote the set union of all geodesics of $H$ that join $x$ to $y$. Then $C_{x y}$ is a closed convex subset of $H$. If $H$ admits no Euclidean de Rham factor, then $C_{x y}$ is always a proper subset of $H$. If $p$ is any point of $C_{x y}$ then

$$
L(p, x) \cap L(p, y)=B(p, x) \cap B(p, y)
$$

is a closed convex subset of $C_{x y}$, and $C_{x y}$ may be naturally identified with the set $\mathbb{R} \times\{L(p, x) \cap L(p, y)\}$.

Proof. We recall from $\S 1$ that $L(p, x), B(p, x)$ denote respectively the horosphere, horoball determined by $p$ and $x$ with similar meanings for $L(p, y), B(p, y)$.

It is obvious that $C_{x y}=\left\{p \in H: \chi_{p}(x, y)=\pi\right\}$. Let $f_{x}, f_{y}$ be arbitrary Busemann functions at $x, y$ and let $f^{*}=f_{x}+f_{y}$. Then $f^{*}$ is a $C^{2}$ convex function by [12, prop. 3.1] and

$$
\left(\operatorname{grad} f^{*}\right)(p)=-V(p, x)-V(p, y) \quad \text { for every point } p
$$

by [8, prop. 3.5] or [4, prop. 2.6]. Recall from $\S 1$ that $V(p, z)=\gamma_{p z}^{\prime}(0)$ for any points $p \in H$ and $z \in H(\infty)$. It follows that $C_{x y}$ is the set of minimum critical points of $f^{*}$, which is a closed convex subset of $H$ since $f^{*}$ is convex. If $H$ has no Euclidean de Rhem factor then $C_{x y}$ is always a proper subset of $H$ by [2, theorem 2.1 and prop. 2.2].

Now let $p$ be any point of $C_{x y}$. The set $B(p, x) \cap B(p, y)$ is a closed convex subset of $H$ since both sets $B(p, x)$ and $B(p, y)$ are closed and convex. We show that

$$
B(p, x) \cap B(p, y)=L(p, x) \cap L(p, y) .
$$

Clearly $L(p, x) \cap L(p, y) \subseteq B(p, x) \cap B(p, y)$ by the definition of these sets and so it suffices to prove the reverse inclusion. Let $q \in B(p, x) \cap B(p, y)$ be given, and let $f^{*}, g^{*}$ be Busemann functions at $x, y$. We may assume that $q \neq p$ and let $\sigma$ denote the geodesic segment $\gamma_{p q}$. Since $f^{*}(q) \leq f^{*}(p)$ by the definition of $q$ it follows that the convex function $f^{*} \circ \sigma$ is non-increasing. Hence

$$
0 \geq\left(f^{*} \circ \sigma\right)^{\prime}(0)=\left\langle\sigma^{\prime}(0), \operatorname{grad} f^{*}(p)\right\rangle=-\left\langle\sigma^{\prime}(0), V(p, x)\right\rangle=-\cos \chi_{p}(q, x) .
$$

It follows that $\chi_{p}(q, x) \leq \pi / 2$ with equality if and only if $\left(f^{*} \circ \sigma\right)^{\prime}(0)=0$. Similarly $\chi_{p}(q, y) \leq \pi / 2$ since $g^{*}(q) \leq g^{*}(p)$ with equality if and only if $\left(g^{*} \circ \sigma\right)^{\prime}(0)=0$. Since 
$p$ lies on a geodesic from $x$ to $y$ it follows that

Therefore

$$
\Varangle_{p}(x, q)+\Varangle_{p}(y, q)=\pi \text {. }
$$

$$
\chi_{p}(q, x)=\chi_{p}(q, y)=\pi / 2
$$

and $\left(f^{*} \circ \sigma\right)^{\prime}(0)=\left(g^{*} \circ \sigma\right)^{\prime}(0)=0$. Since $f^{*} \circ \sigma, g^{*} \circ \sigma$ are non-increasing and $\left(f^{*} \circ \sigma\right)^{\prime \prime} \geq 0,\left(g^{*} \circ \sigma\right)^{\prime \prime} \geq 0$ it follows that $f^{*} \circ \sigma$ and $g^{*} \circ \sigma$ are constant. In particular $f^{*}(p)=f^{*}(q)$ and $g^{*}(p)=g^{*}(q)$, which shows that $q \in L(p, x) \cap L(p, y)$. Therefore

$$
B(p, x) \cap B(p, y) \subseteq L(p, x) \cap L(p, y)
$$

and equality follows immediately.

We show that $B(p, x) \cap B(p, y) \subseteq C_{x y}$ for every point $p \in C_{x y}$. Let $f^{*}$ be a Busemann function at $x$. By [2, lemma 3.2a] it suffices to show that $f^{*}$ is constant on $B(p, x) \cap B(p, y)=L(p, x) \cap L(p, y)$, but by definition $f^{*}$ is constant on all horospheres at $x$.

We conclude the lemma by showing that $C_{x y}$ can be identified with the set $\mathbb{R} \times\{L(p, x) \cap L(p, y)\}$ for any point $p \in C_{x y}$. Let $p \in C_{x y}$ be given, and let

$$
P: H \rightarrow B(p, x) \cap B(p, y)
$$

denote the orthogonal projection. Let $f^{*}$ be the Busemann function at $x$ such that $f^{*}(p)=0$. We define

$$
G: C_{x y} \rightarrow \mathbb{R} \times\{B(p, x) \cap B(p, y)\} \quad \text { by } G(q)=\left(f^{*}(q), P(q)\right) .
$$

We assert that $G$ is a homeomorphism, which will complete the proof of lemma 5.1.

Clearly $G$ is continuous. We assert that $q=\gamma_{q^{*} x}(-t)$ for every $q \in C_{x y}$, where $q^{*}=P(q)$ and $t=f^{*}(q)$. This will prove that $G$ is one-one. Moreover if we define

$$
G^{*}: \mathbb{R} \times\{B(p, x) \cap B(p, y)\} \rightarrow C_{x y} \quad \text { by } G^{*}\left(t, q^{*}\right)=\gamma_{q^{*} x}(-t)
$$

then it will follow immediately that $G^{*}$ is a continuous inverse of $G$. Now let $g^{*}$ be the Busemann function at $y$ such that $g^{*}(p)=0$. It follows that $f^{*}+g^{*} \equiv 0$ on $C_{x y}$ since $C_{x y}$ is the minimum locus of $f^{*}+g^{*}$ by the first part of the proof of lemma 5.1 .

Let $q \in C_{x y}$ be given arbitarily, and let $q^{*}$ be the unique intersection point of $\gamma_{q x}(\mathbb{R})$ with $L(p, x)$. Then $f^{*}\left(q^{*}\right)=0$ since $q^{*} \in L(p, x)$, and hence $g^{*}\left(q^{*}\right)=0$ since $f^{*}+g^{*} \equiv 0$ on $C_{x y}$. Therefore

$$
q^{*} \in L(p, x) \cap L(p, y)=B(p, x) \cap B(p, y) .
$$

If $\tilde{q}$ is any point of $L(p, x) \cap L(p, y) \subseteq L(p, x)$, then $d\left(q^{*}, q\right) \leq d(\tilde{q}, q)$ since $q^{*}$ is the unique point on $L(p, x)$ that is closest to $q$, by [8, prop. 3.2]. Therefore $q^{*}=P(q)$, the foot of $q$ on $B(p, x) \cap B(p, y)=L(p, x) \cap L(p, y)$. By [3, prop. 2.8] it follows that $q=\gamma_{q^{*} x}(-t)$, where $q^{*}=P(q)$ and $t=f^{*}(q)$. The proof of lemma 5.1 is complete.

We begin the proof of proposition 5.1. Recall from $\S 4$ that for $i=1,2$ the set $S_{i}^{*}$ is the set of maximally singular points in $H_{i}^{*}(\infty)$, where $H^{*}=H_{1}^{*} \times H_{2}^{*}$. Fix points $y^{*} \in S_{2}^{*}$ and $p^{*} \in H^{*}$. Let $\gamma_{2}^{*}$ be the geodesic of $H^{*}$ such that $\gamma_{2}^{*}(0)=p^{*}$ and $\gamma_{2}^{*}(\infty)=y^{*}$. By proposition $3.1 \gamma_{2}^{*}$ is maximally singular and by proposition 4.1 
the points $y=\lim _{t \rightarrow+\infty}\left(f \circ \gamma_{2}^{*}\right)(t)$ and $z=\lim _{t \rightarrow+\infty}\left(f \circ \gamma_{2}^{*}\right)(-t)$ exist in $H(\infty)$ and are distinct. Moreover there exists a point $q \in H$ such that $\chi_{q}(y, z)=\pi$. Now let

$$
C=C_{y z}=\left\{q \in H: \Varangle_{q}(y, z)=\pi\right\} \text {. }
$$

Since $H$ has no Euclidean de Rham factor it follows from lemma 5.1 that $C$ is a nonempty, closed, convex, proper subset of $H$.

The function $q \rightarrow d(q, C)$ is a convex function in $H$ by the discussion of $\S 1$, and hence for every positive number $\alpha$ the set

$$
N_{\alpha}(C)=\{q \in H: d(q, C) \leq \alpha\}
$$

is a nonempty closed convex subset of $H$. The set $N_{\alpha}(C)$ is also proper since $q \rightarrow d(q, C)$ cannot be bounded above in $H$ without being constant in $H$. To prove that $B_{p}$ is a proper subset of $H$ for any point $p \in H$ it suffices to show that $B_{p} \subseteq N_{R^{\prime}}(C)$, where

$$
R^{\prime}=R+d\left(p, f p^{*}\right)
$$

and $R>0$ is the constant of proposition 2. By the definition of $B_{p}$ it suffices to show that $\gamma_{p x}(\mathbb{R}) \subseteq N_{R^{\prime}}(C)$ for every $x \in S_{1} \subseteq H(\infty)$, where $S_{1}$ is defined in $\S 4$. By proposition 4.3 it suffices to show that $\gamma_{p x}[0, \infty) \subseteq N_{R^{\prime}}(C)$ for every $x \in S_{1}$. It suffices to consider points $x$ in $\bar{f}\left(S_{1}^{*}\right)$ since $\bar{f}\left(S_{1}^{*}\right)$ is dense in $S_{1}$.

Let $x \in \bar{f}\left(S_{1}^{*}\right)$ and $p \in H$ be given. Let $R^{\prime}=R+d\left(p, f p^{*}\right)$. We show that $\gamma_{p x}[0, \infty) \subseteq N_{R^{\prime}}(C)$. Choose $x^{*} \in S_{1}^{*}$ so that $\tilde{f}\left(x^{*}\right)=x$. Let $\gamma_{1}^{*}$ be the geodesic of $H^{*}$ such that $\gamma_{1}^{*}(0)=p^{*}$ and $\gamma_{1}^{*}(\infty)=x^{*}$. By propositions 3.1 and $4.1, \gamma_{1}^{*}$ is maximally singular in $H^{*}$ and the points $x=\lim _{t \rightarrow+\infty}\left(f \circ \gamma_{1}^{*}\right)(t)$, $w=\lim _{t \rightarrow+\infty}\left(f \circ \gamma_{1}^{*}\right)(-t)$ exist and can be joined by a geodesic of $H$.

The first step in proving that $\gamma_{p x}[0, \infty) \subseteq N_{R^{\prime}}(C)$ is to show that there exists in $H$ an $r$-flat $F$, where $r$ is the rank of $H^{*}$, such that $F \subseteq C=C_{y z}$ and $\chi_{q}(x, w)=\pi$ for every point $q \in F$. Since $H^{*}=H_{1}^{*} \times H_{2}^{*}$ we may write $p^{*}=\left(p_{1}^{*}, p_{2}^{*}\right)$ where $p_{i}^{*} \in H_{i}^{*}$ for $i=1,2$. The geodesic $\gamma_{1}^{*}$ may be identified with a geodesic of $H_{1}^{*}$ that starts at $p_{1}^{*}$ since $\gamma_{1}^{*}(\infty)=x^{*} \in H_{1}^{*}(\infty)$. Similarly the geodesic $\gamma_{2}^{*}$ defined earlier may be identified with a geodesic of $H_{2}^{*}$ that starts at $p_{2}^{*}$. For $i=1,2$ let $r_{i}$ denote the rank of $H_{i}^{*}$, and let $F_{i}^{*}$ be an $r_{i}$-flat that contains $\gamma_{i}^{*}(\mathbb{R})$. Note that $r=r_{1}+r_{2}$. If $F^{*}=F_{1}^{*} \times F_{2}^{*}$ then $F^{*}$ is an $r$-flat in $H^{*}$ that contains both $\gamma_{1}^{*}(\mathbb{R})$ and $\gamma_{2}^{*}(\mathbb{R})$. By proposition 2 we can find an $r$-flat $F$ of $H$ such that $\mathrm{Hd}\left(f\left(F^{*}\right), F\right) \leq R$. By proposition 4.1 we have

$$
\Varangle_{q}(y, z)=\Varangle_{q}(x, w)=\pi
$$

for every point $q \in F$, which shows that $F \subseteq C=C_{y z}$.

We show next that

$$
d(p, F) \leq R^{\prime}=R+d\left(p, f p^{*}\right) .
$$

Let $P: H \rightarrow F$ denote the orthogonal projection, and let $q=P\left(f p^{*}\right)$. Then

$$
\begin{aligned}
d(p, F) & \leq d(p, q) \\
& \leq d\left(p, f p^{*}\right)+d\left(f p^{*}, q\right)=d\left(p, f p^{*}\right)+d\left(f p^{*}, F\right) \\
& \leq d\left(p, f p^{*}\right)+\mathrm{Hd}\left(\mathrm{f}\left(\mathrm{F}^{*}\right), \mathrm{F}\right) \\
& \leq \mathrm{d}\left(\mathrm{p}, \mathrm{fp}^{*}\right)+\mathrm{R}=\mathrm{R}^{\prime} .
\end{aligned}
$$


Finally we show that $\gamma_{p x}[0, \infty) \subseteq N_{R^{\prime}}(C)$. Let $\sigma(t)=\gamma_{r x}(t)$, where $r=P(p) \in F$. Note that $\sigma(\mathbb{R}) \subseteq F$ by proposition 4.1. Recall that the function $t \rightarrow d\left(\gamma_{p x} t, \sigma(\mathbb{R})\right)$ is convex and bounded above for $t \geq 0$. Hence this function is non-increasing in $t$. Since $F \subseteq C$ it follows that for every $t \geq 0$ we have

$$
\begin{aligned}
d\left(\gamma_{p x} t, C\right) & \leq d\left(\gamma_{p x} t, F\right) \leq d\left(\gamma_{p x} t, \sigma(\mathbb{R})\right) \\
& \leq d\left(\gamma_{p x} 0, \sigma(\mathbb{R})\right)=d(p, \sigma(\mathbb{R})) \leq d(p, r)=d(p, F) \leq R^{\prime} .
\end{aligned}
$$

Therefore $\gamma_{p \times}[0, \infty) \subseteq N_{R^{\prime}}(C)$, which completes the proof of proposition 5.1.

Proof of proposition 5.4. We first need the following:

LEMMA 5.4. For every point $p \in H$ there exists a number $A>0$, possibly depending on $p$, such that if $r \in B_{p}$ is given arbitrarily then $d\left(p, B_{r}\right) \leq A$.

Assuming for the moment that the lemma has been proved we prove the proposition 5.4. Let $p$ be any point of $H$ and let $A>0$ be chosen as in the lemma above. Let $h: B_{p} \rightarrow \mathbb{R}$ be given by $h(r)=d\left(p, B_{r}\right)$. We shall show that $h$ has a maximum value at some point $r^{*} \in B_{p}$. If $r^{* *}$ is the foot of $p$ on $B_{r^{*}}$ we then show that $B_{r^{* *}}$ is a minimally convex set. We show first that $h$ is continuous. It suffices to prove that

$$
|h(r)-h(s)| \leq d(r, s)
$$

for all points $r, s$ in $B_{p}$. Let $r, s$ be given in $B_{p}$. Let $r^{*}$ denote the foot of $p$ on $B_{n}$ and let $s^{*}$ denote the foot of $r^{*}$ on $B_{s}$. Then

$$
\begin{aligned}
d\left(p, B_{s}\right) & \leq d\left(p, s^{*}\right) \leq d\left(p, r^{*}\right)+d\left(r^{*}, s^{*}\right)=d\left(p, B_{r}\right)+d\left(r^{*}, B_{s}\right) \\
& \leq d\left(p, B_{r}\right)+d\left(r, B_{s}\right) \leq d\left(p, B_{r}\right)+d(r, s) .
\end{aligned}
$$

In the second to last inequality above we apply lemma $5.5 \mathrm{a}$, which is proved later in this appendix, to the points $r$ and $r^{*} \in B_{r}$. The inequalities above show that

$$
d\left(p, B_{s}\right)-d\left(p, B_{r}\right) \leq d(r, s) .
$$

Reversing the roles of $r$ and $s$ we obtain

$$
d\left(p, B_{r}\right)-d\left(p, B_{s}\right) \leq d(s, r),
$$

which proves $\left|d\left(p, B_{r}\right)-d\left(p, B_{s}\right)\right| \leq d(r, s)$.

By the previous paragraph and lemma 5.4 it follows that $h$ is continuous and bounded above on $B_{p}$. We show that $h$ has a maximum value on $B_{p}$. Let $\left\{r_{n}\right\} \subseteq B_{p}$ be a sequence such that

$$
h\left(r_{n}\right) \rightarrow \sup h \leq A \quad \text { as } n \rightarrow+\infty .
$$

Let $r_{n}^{*}$ be the foot of $p$ on $B_{r_{n}}$. By proposition $5.2 B_{r_{n}^{*}} \subseteq B_{r_{n}}$ and hence

$$
d\left(p, B_{r_{n}^{*}}\right) \geq d\left(p, B_{r_{n}}\right) \quad \text { for every } n .
$$

It follows that $h\left(r_{n}^{*}\right) \rightarrow \sup h$ as $n \rightarrow+\infty$. Moreover

$$
d\left(p, r_{n}^{*}\right)=d\left(p, B_{r_{n}}\right) \leq A \quad \text { for every } n,
$$

where $A>0$ is the constant of lemma 5.4. Passing to a subsequence we may let $\left\{r_{n}^{*}\right\}$ converge to a point $r^{*} \in B_{p}$. By the continuity of $h$ we see that $h\left(r^{*}\right)=\sup h$.

Finally let $r^{* *}$ be the foot of $p$ on $B_{r^{*}}$. We assert that $B_{r^{* *}}$ is minimally convex. Let $q$ be an arbitrary point of $B_{r^{* *}}$, and let $q^{*} \in B_{q}$ be the foot of $p$ on $B_{q}$, Since $h$ 
has its maximum value at $r^{*}$ it follows that

$$
d\left(p, q^{*}\right)=d\left(p, B_{q}\right) \leq d\left(p, B_{r^{*}}\right)=d\left(p, r^{* *}\right) .
$$

By proposition 5.2 we have $B_{q} \subseteq B_{r^{* *}} \subseteq B_{r^{*}}$. In particular $q^{*} \in B_{r^{*}}$. Since $d\left(p, q^{*}\right) \leq$ $d\left(p, r^{* *}\right)$ as we showed above and since $r^{* *}$ is the unique point in $B_{r^{*}}$ that is closest to $p(\& 1)$ it follows that $r^{* *}=q^{*} \in B_{q}$. Therefore $B_{r^{* *}} \subseteq B_{q}$ by proposition 5.2. We also know from proposition 5.2 that $B_{q} \subseteq B_{r * *}$ since $q$ is defined to be a point of $B_{r * *}$. Therefore $B_{q}=B_{r * *}$, which shows that $B_{r^{* *}}$ is minimally convex.

We now prove lemma 5.4 .

SublemMA 5.4a. Let $H$ be an arbitrary Hadamard manifold, and let $x$ be any point of $H(\infty)$. Let $Z$ denote the unit vector field in $H$ given by

$$
Z(p)=V(p, x)=\gamma_{p x}^{\prime}(0) .
$$

Let $\gamma:[0, c] \rightarrow H$ be a unit speed geodesic such that

$$
\Varangle\left(\gamma^{\prime} t, Z(\gamma t)\right) \equiv \theta>0 \quad \text { for every } t \in[0, c] .
$$

If $D=[0, \infty) \times[0, c]$ we define $F: D \rightarrow H$ by

$$
F(s, t)=\exp _{\gamma t}(s Z(\gamma t)) \text {. }
$$

Then $F(D)$ is a closed, flat, totally geodesic imbedded surface with boundary in $H$.

SublemMA 5.4b. Let $\mathrm{H}$ be a Hadamard manifold satisfying the hypotheses of theorem $A$ and let $\gamma$ be a maximal geodesic of $H$ such that $\gamma[0, \infty) \subseteq B_{p}$, where $p=\gamma(0)$. Let $z=\gamma(\infty)$. Then there exists $x \in S_{1}$ and $t \in \mathbb{R}$ such that $\chi_{\gamma^{t}}(x, z) \neq \pi / 2$.

Assuming for the moment that the sublemmas have been proved we begin the proof of lemma 5.4. Since the proof is rather long we give an outline. Suppose that lemma 5.4 is false for some point $p \in H$, and let $\left\{q_{n}\right\} \subseteq B_{p}$ be a sequence such that

$$
d\left(p, B_{q_{n}}\right)=r_{n} \rightarrow+\infty \quad \text { as } n \rightarrow+\infty \text {. }
$$

Let $q_{n}^{*}$ be the foot of $p$ on $B_{q_{n}}$, and let $\left\{q_{n}^{*}\right\}$ converge to a point $z \in H(\infty)$ by passing to a subsequence. If $\gamma(t)=\gamma_{p z}(t)$ we show that $\chi_{\gamma t}(x, z)=\pi / 2$ for all $t \geq 0$ and for all $x \in S_{1}$. Moreover if $B_{t}$ denotes $B_{\gamma(t)}$ for $t \geq 0$ then $B_{t}$ contains $\gamma[t, \infty)$ for every $t \geq 0$. Now choose a sequence $\left\{\phi_{n}\right\} \subseteq \Gamma$, the deckgroup of the compact manifold $M=H / \Gamma$, so that, by passing to a subsequence if necessary, $p_{n}^{*}=$ $\left(\phi_{n} \circ \gamma\right)(n)$ converges to a point $p^{*} \in H$ and $\phi_{n} z$ converges to a point $z^{*} \in H(\infty)$. If $\beta=\gamma_{p^{*} z^{*}}$ then $\beta[0, \infty) \subseteq B_{p^{*}}$ and $\chi_{\beta t}\left(x, z^{*}\right)=\pi / 2$ for every $x \in S_{1}$ and every $t \in \mathbb{R}$. This will contradict sublemma $5.4 \mathrm{~b}$ and will complete the proof of lemma 5.4 .

We now fill in the details. Suppose that lemma 5.4 is false for some point $p \in H$ and let $\left\{q_{n}\right\} \subseteq B_{p},\left\{q_{n}^{*}\right\} \subseteq H$ and $z \in H(\infty)$ be defined as above. By lemma 5.5a, proved later in this section, the geodesics $\gamma_{p x}(\mathbb{R})$ and $\gamma_{q_{n x}^{*}}(\mathbb{R})$ bound a flat strip $F_{n}$ in $H$ for every $n, x \in S_{1}$ (See $[8, \S 5]$ or $[9$, Cor. 4.2] for a definition and discussion of flat strips.) Hence if $\gamma_{n}$ denotes the unit speed geodesic $\gamma_{p q_{n}^{*}}$ then by the Euclidean geometry of $F_{n}$ we have

$$
\Varangle_{\gamma_{n} t}\left(\gamma_{n}(\infty), x\right)=\pi / 2 \quad \text { for all } t \in\left[0, t_{n}\right],
$$


where $t_{n}=d\left(p, q_{n}^{*}\right) \rightarrow+\infty$ as $n \rightarrow+\infty$. By continuity it follows that $\mathrm{X}_{\gamma t}(z, x)=\pi / 2$ for all $t \geq 0$ and all $x \in S_{1}$ where $\gamma(t)=\gamma_{p z}(t)$.

Next we prove that $B_{t}=B_{\gamma(t)}$ contains $\gamma[t, \infty)$ for every $t \geq 0$. We show first that $\gamma[0, \infty) \subseteq B_{p}$. Since $q_{n}^{*} \in B_{q_{n}} \subseteq B_{p}$ it follows that $\gamma_{n}\left[0, t_{n}\right] \subseteq B_{p}$ for every $n$ by the convexity of $B_{p}$. Then since $\gamma_{n}^{\prime}(0) \rightarrow \gamma^{\prime}(0), t_{n} \rightarrow+\infty$ and $B_{p}$ is closed it follows that $\gamma[0, \infty) \subseteq B_{p}$. Now fix $t \geq 0$ and let $s \geq t$ be given arbitrarily. Let $\xi(s)$ be the foot of $\gamma(s)$ on $B_{t}$. By lemma 5.5a below we have

$$
d(\gamma s, \xi s)=d\left(\gamma s, B_{t}\right) \leq d\left(p, B_{t}\right)
$$

a constant independent of $s$. Since this inequality holds for every $s \geq t$ we obtain

$$
\lim _{s \rightarrow+\infty} \xi(s)=\lim _{s \rightarrow+\infty} \gamma(s)=z .
$$

For every $s \geq t$ the geodesic segment from $\gamma(t)$ to $\xi(s)$ is contained in $B_{t}$ by the convexity of $B_{t}$. Letting $s \rightarrow+\infty$ it follows that $\gamma[t, \infty) \subseteq B_{t}$ by continuity and the fact that $B_{t}$ is closed.

Since $M=H / \Gamma$ is compact there exists a sequence $\left\{\phi_{n}\right\} \subseteq \Gamma$ so that $p_{n}^{*}=\left(\phi_{n} \circ \gamma\right)(n)$ is a bounded sequence in $H$. Define

$$
\beta_{n}(t)=\left(\phi_{n} \circ \gamma\right)(t+n) \text {. }
$$

Since $S_{1}$ is invariant under $\Gamma$ it follows from the work above that for every $n$ we have $\chi_{\beta_{n^{\prime}}}\left(x, \phi_{n} z\right)=\pi / 2$ for every $x \in S_{1}$ and every $t \geq-n$. Moreover

$$
\beta_{n}[0, \infty) \subseteq \phi_{n}\left(B_{\gamma(n)}\right)=B_{p_{n}^{*}} \quad \text { for every } n .
$$

Passing to a subsequence let $p_{n}^{*}=\beta_{n}(0)$ converge to a point $p^{*} \in H$ and let $\phi_{n} z$ converge to a point $z^{*} \in H(\infty)$. If $\beta=\gamma_{p^{*} z^{*}}$ then $\beta_{n}^{\prime}(0) \rightarrow \beta^{\prime}(0)$ as $n \rightarrow+\infty$. By continuity it follows that $\chi_{\beta t}\left(x, z^{*}\right)=\pi / 2$ for every $x \in S_{1}$ and for every $t \in \mathbb{R}$.

To obtain a contradiction to sublemma $5.4 \mathrm{~b}$ and complete the proof of lemma 5.4 it only remains to show that $\beta[0, \infty) \subseteq B_{p}^{*}$. Let $t \geq 0$ be given and let $a_{n}$ be the foot of $\beta_{n} t$ on $B_{p^{*}}$. Since $\beta_{n}[0, \infty) \subseteq B_{p_{n}^{*}}$ it follows from lemma 5.5a below that

$$
d\left(\beta_{n} t, a_{n}\right)=d\left(\beta_{n} t, B_{p^{*}}\right) \leq d\left(p_{n}^{*}, B_{p}^{*}\right) \leq d\left(p_{n}^{*}, p^{*}\right) \rightarrow 0 \quad \text { as } n \rightarrow+\infty .
$$

Therefore $\lim _{n \rightarrow \infty} a_{n}=\lim _{n \rightarrow \infty} \beta_{n} t=\beta t$. Hence $\beta t \in B_{p}^{*}$ for every $t \geq 0$ since $B_{p^{*}}$ is closed.

We now prove the two sublemmas beginning with 5.4a. We adopt the notation in the statement of sublemma 5.4a. We show first that $F: D \rightarrow H$ is a $C^{1}$ imbedding. The vector field $Z$ is $C^{1}$ by [12, prop. 3.1], and hence $F$ is $C^{1}$. It is clear that $F$ is one-one and $F(D)$ is a closed subset of $H$. It remains to show that $F$ is nonsingular on $D$. Let $r_{s}(s, t)$ and $r_{t}(s, t)$ denote respectively $F_{*} \partial / \partial s(s, t)$ and $F_{*} \partial / \partial t(s, t)$. Note that $\left\|r_{s}(s, t)\right\| \equiv 1$ in $D$ since the $s$-parameter curves are geodesics of $H$. From [12, prop. 3.2] it follows that $r_{t}(s, t)$ is never zero and never collinear with $r_{s}(s, t)$. Hence $F$ is nonsingular on $D$.

We show next that $t \rightarrow Z(\gamma t)$ is a parallel vector field on $\gamma[0, c]$ or equivalently that $\left(\nabla_{r_{t}} r_{s}\right)(0, t) \equiv 0$ in $[0, c]$. Here $\nabla$ denotes covariant differentiation in $H$ and $\left(\nabla_{r_{t}} r_{s}\right)(0, t)$ is shorthand for the covariant derivative of $t \rightarrow r_{s}(0, t)$ along the curve $t \rightarrow F(0, t)$. The fact that $t \rightarrow Z(\gamma t)$ is a parallel vector field will show that it is $C^{\infty}$ and will imply that $F: D \rightarrow H$ is actually a $C^{\infty}$ imbedding. 
Let $f^{*}$ be a Busemann function at $x$. The function $f^{*}$ is $C^{2}$ and convex by [12, prop. 3.1] and $\operatorname{grad} f^{*}=-Z$ by [8, prop. 3.5] or [4, prop. 2.6]. The bilinear form

$$
(v, w) \rightarrow\left(\nabla^{2} f^{*}\right)(v, w)=\left\langle\nabla_{v} \operatorname{grad} f^{*}, w\right\rangle
$$

is symmetric for any $C^{2}$ function $f^{*}$ and the convexity of $f^{*}$ is equivalent to the condition that this form be positive semidefinite. By hypothesis

$$
\begin{aligned}
\cos \theta & \equiv \cos \left(\chi\left(\gamma^{\prime} t, Z(\gamma t)\right)\right)=\left\langle\gamma^{\prime} t, Z(\gamma t)\right\rangle \\
& =-\left\langle\gamma^{\prime}(t), \operatorname{grad} f^{*}(\gamma t)\right\rangle=-\left(f^{*} \circ \gamma\right)^{\prime}(t)
\end{aligned}
$$

for every $t \in[0, c]$. Hence

$$
0 \equiv\left(f^{*} \circ \gamma\right)^{\prime \prime}(t)=\left\langle\nabla_{\gamma^{\prime} t} \operatorname{grad} f^{*}, \gamma^{\prime} t\right\rangle
$$

for every $t \in[0, c]$. It follows that

$$
\nabla_{\gamma^{\prime} t} Z=-\nabla_{\gamma^{\prime} t} \operatorname{grad} f^{*} \equiv 0 \quad \text { on }[0, c]
$$

since $\nabla^{2} f^{*}$ is positive semidefinite and symmetric.

Now let $g: D \rightarrow \mathbb{R}$ be given by $g(s, t)=\left\|r_{t}(s, t)\right\|^{2}$. Since the curves $\rightarrow F(s, t)$ are unit speed geodesics it is routine to compute that

$$
\partial g / \partial s=2\left\langle\nabla_{r_{s}} r_{t}, r_{t}\right\rangle
$$

and

$$
\partial^{2} g / \partial s^{2}=2\left\{\left\|\nabla_{r_{s}} r_{t}\right\|^{2}-\left\langle R\left(r_{s}, r_{t}\right) r_{s}, r_{t}\right\rangle\right\} \geq 0,
$$

where $R$ denotes the curvature tensor of $H$. The fact that $\nabla_{\gamma^{\prime} t} Z \equiv 0$ in $[0, c]$ means that $(\partial g / \partial s)(0, t) \equiv 0$ in $[0, c]$. Since $\partial^{2} g / \partial s^{2} \geq 0$ it follows that $\partial g / \partial s \geq 0$ for $s \geq 0$ and $s \rightarrow g(s, t)$ is non-decreasing on $[0, \infty)$ for each $t \in[0, c]$. On the other hand it follows from [12, prop. 3.2] that $s \rightarrow g(s, t)$ is non-increasing on $[0, \infty)$ since $s \rightarrow r_{t}(s, t)$ is a stable Jacobi vector field on the geodesic $s \rightarrow F(s, t)$. Hence $g$ is constant in $D$ and $\partial^{2} g / \partial s^{2}$ is identically zero on $D=[0, \infty) \times[0, c]$. From the expression for $\partial^{2} g / \partial s^{2}$ it follows that

$$
\nabla_{r_{s}} r_{t} \equiv 0 \text { and }\left\langle R\left(r_{s}, r_{t}\right) r_{s}, r_{t}\right\rangle \equiv 0 \quad \text { in } D .
$$

To show that $F$ is totally geodesic it suffices to show that $\nabla_{r_{t}} r_{t} \equiv 0$ in $D$, since $\nabla_{r_{s}} r_{s} \equiv 0$ in $D$ by the fact that the $s$-parameter curves of $F$ are unit speed geodesics of $H$. It will then follow that $F(D)$ is flat by the curvature equality above, and this will complete the proof of the sublemma.

Define $h(s, t)=\left\|\nabla_{r_{t}} r_{t}\right\|^{2}(s, t)$. We compute

$$
\begin{aligned}
\partial h / \partial s & =2\left\langle\nabla_{r_{s}}\left(\nabla_{r_{t}} r_{t}\right), \nabla_{r_{t}} r_{t}\right\rangle \\
& =2\left\langle\nabla_{r_{t}}\left(\nabla_{r_{s}} r_{t}\right), \nabla_{r_{t}} r_{t}\right\rangle+2\left\langle R\left(r_{t}, r_{s}\right) r_{t}, \nabla_{r_{t}} r_{t}\right\rangle .
\end{aligned}
$$

The first term vanishes since $\nabla_{r_{s}} r_{t} \equiv 0$. The second term vanishes since $R\left(r_{t}, r_{s}\right) r_{t} \equiv 0$; this follows since the linear transformation $v \rightarrow R\left(r_{t}, v\right) r_{t}$ is symmetric and negative semidefinite, and $\left\langle R\left(r_{t}, r_{s}\right) r_{t}, r_{s}\right\rangle \equiv 0$. Therefore $\partial h / \partial s \equiv 0$ in $D$. We recall that $h(0, t) \equiv$ 0 on $[0, c]$ since $t \rightarrow F(0, t)=\gamma(t)$ is a geodesic of $H$. Therefore $h \equiv 0$ on $D$, which proves that $F$ is totally geodesic and completes the proof of sublemma $5.4 \mathrm{a}$.

We prove sublemma 5.4b. Suppose that the sublemma is false. Then we can find a maximal geodesic $\gamma$ in $H$ such that $\gamma[0, \infty) \subseteq B_{p}$, where $p=\gamma(0)$, and $\Varangle_{\gamma t}(x, z)=$ 
$\pi / 2$ for every $t \in \mathbb{R}$ and every $x \in S_{1}$, where $z=\gamma(\infty)$. If $x \in S_{1}$ is fixed, then by sublemma 5.4a the set $F=F([0, \infty) \times \mathbb{R})$ is a closed, totally geodesic flat half-plane whose boundary is $\gamma(\mathbb{R})$. Moreover $\gamma_{p x}[0, \infty) \subseteq F$. Since $\gamma_{p x}$ meets $\gamma$ orthogonally at $p$ it follows from lemma 5.1 and the Euclidean geometry of $F$ that $\gamma_{p x}[0, \infty)$ is contained in the proper, closed, convex subset $L(p, z) \cap L(p, w)=$ $B(p, z) \cap B(p, w)$, where $w=\gamma(-\infty)$. By proposition 4.3 we know that $\gamma_{p x}(-\infty) \in S_{1}$, and hence

$$
\gamma_{p x}(-\infty, 0] \subseteq B(p, z) \cap B(p, w)
$$

by the argument above. It follows that every geodesic $\gamma_{p x}(\mathbb{R})$ is contained in $B(p, z) \cap B(p, w)=L(p, z) \cap L(p, w)$ as $x$ ranges over $S_{1}$. Since $B_{p}$ is the closed convex hull of these geodesics it follows that $B_{p} \subseteq L(p, z) \cap L(p, w) \subseteq L(p, z)$. However, by hypothesis $\gamma[0, \infty) \subseteq B_{p}$, which contradicts the fact that $\gamma(\mathbb{R})$ meets $L(p, z)$ exactly once at the point $p[\mathbf{8}, \mathrm{p} .57]$. This contradiction proves sublemma $5.4 \mathrm{~b}$, and this completes the proof of proposition 5.4.

Proof of the convexity of $\tilde{H}$ in proposition 5.5. The idea of the proof is simple but the technical details are annoying. Let $r$ be an interior point of a geodesic segment joining two points $p, q$ of $\tilde{H}$. Let $p^{\prime}$ be the foot of $r$ on $B_{p}$. Let $r^{*}$ be an arbitrary point of $B_{r}$, and let $\tilde{r}$ be the foot of $p^{\prime}$ on $B_{r^{*}}$. We show that $r=\tilde{r}$ by showing that both points lie on the perpendicular from $p^{\prime}$ to $B_{q}$ at equal distances from $p^{\prime}$. It then follows from proposition 5.2 that $B_{r}=B_{r}^{*}$ and hence $r \in \tilde{H}$. We need the following two lemmas:

LEMMA 5.5a. Let $p, r$ be any two points of $H$. Then $d\left(q, B_{r}\right) \leq d\left(p, B_{r}\right)$ for every point $q \in B_{p}$. Moreover suppose that $p \notin B_{r}$, and let $P: H \rightarrow B_{r}$ denote the orthogonal projection. Let $p^{*}=P(p)$. Then for every $x \in S_{1}$ we have

$$
\gamma_{p^{*} x}(t)=\left(P \circ \gamma_{p x}\right)(t) \quad \text { for every } t,
$$

and $\gamma_{p^{*} x}(\mathbb{R}), \gamma_{p x}(\mathbb{R})$ bound a flat strip in $H$.

LEMMA 5.5b. Let $p, q$ be points of $H$ such that $B_{p}, B_{q}$ are distinct minimally convex sets. Then $\xi \rightarrow d\left(\xi, B_{q}\right)$ and $\eta \rightarrow d\left(\eta, B_{p}\right)$ are constant functions on $B_{p}$ and $B_{q}$ respectively. Moreover any perpendicular from $B_{p}$ to $B_{q}$ or from $B_{q}$ to $B_{p}$ is a mutual perpendicular.

For the moment we defer the proofs of the two lemmas and begin the proof of the convexity of $\tilde{H}$. Let $p, q$ be distinct points of $\tilde{H}$, and let $r$ be an interior point of the geodesic segment from $p$ to $q$. We may assume that $p \notin B_{q}$ and $q \notin B_{p}$ for otherwise $B_{r}=B_{p}=B_{q}$ by the minimality of the sets $B_{p}, B_{q}$ and this implies that $r \in \tilde{H}$. The first step is to show that $r$ lies on a mutual perpendicular between $B_{p}$ and $B_{q}$. Let $p^{*}$ denote the foot of $q$ on $B_{p}$, and let $q^{*}$ denote the foot of $p$ on $B_{q}$. If $P: H \rightarrow B_{q}$ denotes the orthogonal projection, then $P\left(p^{*}\right)=q$ since the perpendicular from $q$ to $B_{p}$ is also a perpendicular from $p^{*}$ to $B_{q}$ by lemma $5.5 \mathrm{~b}$. Hence

$$
d\left(q, q^{*}\right)=d\left(P p^{*}, P p\right) \leq d\left(p^{*}, p\right)
$$

by [1, lemma 3.2]. Reversing the roles of $B_{p}$ and $B_{q}$ we obtain $d\left(p, p^{*}\right) \leq d\left(q, q^{*}\right)$. 
Hence

$$
d\left(p, p^{*}\right)=d\left(q, q^{*}\right)=d\left(P p^{*}, P p\right)
$$

and by $[1$, remark 3.3, p. 8$]$ we see that $p, p^{*}, q, q^{*}$ are the vertices of an imbedded, flat, totally geodesic plane rectangle $R^{*}$ in $H$. Therefore $r$ lies on a geodesic segment $\sigma$ in $R^{*}$ of length

$$
a=d\left(p, q^{*}\right)=d\left(q, p^{*}\right)=d\left(p, B_{q}\right)=d\left(q, B_{p}\right),
$$

and the endpoints $p^{\prime}, q^{\prime}$ of $\sigma$ lie on the geodesic segments $\gamma_{p p^{*}}, \gamma_{q q^{*}}$. Hence $p^{\prime} \in B_{p}$ and $q^{\prime} \in B_{q}$ by the convexity of $B_{p}, B_{q}$. Finally

$$
a=d\left(p^{\prime}, B_{q}\right) \leq d\left(p^{\prime}, q^{\prime}\right)=L(\sigma)=a,
$$

with the first equality coming from lemma 5.5b. It follows that $d\left(p^{\prime}, q^{\prime}\right)=d\left(p^{\prime}, B_{q}\right)$, which means that $q^{\prime}$ is the foot of $p^{\prime}$ on $B_{q}$. Similarly one can show that $p^{\prime}$ is the foot of $q^{\prime}$ on $B_{p}$. Therefore $r$ lies on a mutual perpendicular $\sigma$ between $B_{p}$ and $B_{q}$.

For the remainder of the proof $p^{\prime}$ and $q^{\prime}$ will retain their meaning of the paragraph above. The next step is to show that

$\left(^{*}\right)$ the functions $\xi \rightarrow d\left(\xi, B_{p}\right)$ and $\xi \rightarrow d\left(\xi, B_{q}\right)$ are constant on $B_{r}$.

Let $r^{*} \in B$, be given, and let $\bar{p}, \bar{q}$ be the feet of $r^{*}$ on $B_{p}, B_{q}$ respectively. Then

(1) $d\left(r^{*}, \bar{p}\right)=d\left(r^{*}, B_{p}\right) \leq d\left(r, B_{p}\right)=d\left(r, p^{\prime}\right) \quad$ by lemma $5.5 \mathrm{a}$.

Similarly we have

(2) $d\left(r^{*}, \bar{q}\right)=d\left(r^{*}, B_{q}\right) \leq d\left(r, B_{q}\right)=d\left(r, q^{\prime}\right)$

Hence

$$
\begin{aligned}
a & =d\left(\bar{p}, B_{q}\right) \\
& \leq d(\bar{p}, \bar{q}) \leq d\left(\bar{p}, r^{*}\right)+d\left(r^{*}, \bar{q}\right) \\
& \leq d\left(p^{\prime}, r\right)+d\left(r, q^{\prime}\right) \\
& =d\left(p^{\prime}, q^{\prime}\right)=a
\end{aligned}
$$

by lemma $5.5 b$

by (1) and (2)

since $p^{\prime}, r, q^{\prime}$ are collinear

Therefore all inequalities above are equalities. From the third inequality it follows that $d\left(\bar{p}, r^{*}\right)=d\left(r, p^{\prime}\right)$ and $d\left(r^{*}, \bar{q}\right)=d\left(r, q^{\prime}\right)$; that is, equality holds in (1) and (2) above. Therefore $d\left(r^{*}, B_{p}\right)=d\left(r, B_{p}\right)$ and $d\left(r^{*}, B_{q}\right)=d\left(r, B_{q}\right)$, which proves $\left(^{*}\right)$.

We reach the last step in the proof that $\tilde{H}$ is convex. Let $r^{*}$ be an arbitrary point of $B_{r}$, and let $\tilde{r}$ be the foot of $p^{\prime}$ on $B_{r^{*}}$. We show that $\tilde{r}=r$, which will show that $B_{r^{*}}=B_{r}$ and $r \in \tilde{H}$ as we explained earlier. Let $p^{\prime \prime}$ be the foot of $\tilde{r}$ on $B_{p}$, and let $q^{\prime \prime}$ be the foot of $\tilde{r}$ on $B_{q}$. Then

$$
\begin{aligned}
d\left(p^{\prime}, B_{q}\right) & \leq d\left(p^{\prime}, q^{\prime \prime}\right) \leq d\left(p^{\prime}, \tilde{r}\right)+d\left(\tilde{r}, q^{\prime \prime}\right)=d\left(p^{\prime}, B_{r^{*}}\right)+d\left(\tilde{r}, B_{q}\right) \\
& \leq d\left(p^{\prime \prime}, B_{r^{*}}\right)+d\left(r, B_{q}\right) \quad \text { by }\left(^{*}\right) \text { above, lemma 5.5a and since } p^{\prime} \in B_{p} \\
=B_{p^{\prime \prime}} & \\
& \leq d\left(p^{\prime \prime}, \tilde{r}\right)+d\left(r, q^{\prime}\right)=d\left(\tilde{r}, B_{p}\right)+d\left(r, q^{\prime}\right) \\
& =d\left(r, B_{p}\right)+d\left(r, q^{\prime}\right)=d\left(r, p^{\prime}\right)+d\left(r, q^{\prime}\right) \quad \text { by }\left(^{*}\right) \text { above } \\
& =d\left(p^{\prime}, q^{\prime}\right)=d\left(p^{\prime}, B_{q}\right) \quad \text { since } p^{\prime}, r, q^{\prime} \text { are collinear. }
\end{aligned}
$$

Hence all inequalities above are equalities. From the first inequality we see that $q^{\prime \prime}=q^{\prime}$, the foot of $p^{\prime}$ on $B_{q}$. From the second inequality we see that $p^{\prime}, \tilde{r}$ and $q^{\prime \prime}=q^{\prime}$ are collinear. Recall that $p^{\prime}, r$ and $q^{\prime}$ are also collinear by work above. To prove that $\tilde{r}=r$ it suffices to prove that $d\left(p^{\prime}, \tilde{r}\right)=d\left(p^{\prime}, r\right)$. 
From the inequalities above it follows that $d\left(p^{\prime}, \tilde{r}\right)=d\left(p^{\prime \prime}, \tilde{r}\right)$ and from the condition $\left({ }^{*}\right)$ above we see that

$$
d\left(p^{\prime \prime}, \tilde{r}\right)=d\left(\tilde{r}, B_{p}\right)=d\left(r, B_{p}\right)=d\left(r, p^{\prime}\right) .
$$

Hence $d\left(p^{\prime}, \tilde{r}\right)=d\left(p^{\prime}, r\right)$, which proves that $\tilde{r}=r$ and completes the proof that $\tilde{H}$ is convex.

We now prove lemmas $5.5 \mathrm{a}$ and $5.5 \mathrm{~b}$, beginning with $5.5 \mathrm{a}$. Let $p, r$ be any two points of $H$, and let $q$ be any point of $B_{p}$. If $p \in B_{r}$ then $B_{q} \subseteq B_{p} \subseteq B_{r}$ by proposition 5.2 and $d\left(q, B_{r}\right)=d\left(p, B_{r}\right)=0$. We may therefore assume that $p \notin B_{r}$. Let $p^{*} \in B_{r}$ be the foot of $p$ on $B_{r}$, and let $x \in S_{1}$ be given arbitrarily. Let $P: H \rightarrow B_{r}$ denote the orthogonal projection.

We show first that $\left(P \circ \gamma_{p x}\right)(t)=\gamma_{p^{*} x}(t)$ for every $t \in \mathbb{R}$. By proposition 5.2 and the definition of $B_{p^{*}}$ we see that $\gamma_{p^{*} x}(\mathbb{R}) \subseteq B_{p^{*}} \subseteq B_{r}$. Similarly $\gamma_{p x}(\mathbb{R}) \subseteq B_{p}$. The function $t \rightarrow d^{2}\left(\gamma_{p x} t, \gamma_{p^{*} x}\right)$ is convex and bounded above for $t \geq 0$ by [1, Theorem 4.1] and the fact that $\gamma_{p x}$ and $\gamma_{p^{*} x}$ are asymptotes. Hence $t \rightarrow d\left(\gamma_{p x} t, \gamma_{p^{*} x}\right)$ is nonincreasing in $t$. For every $t \geq 0$ we obtain

$$
\begin{aligned}
d\left(\gamma_{p x} t, B_{r}\right) & \leq d\left(\gamma_{p x} t, \gamma_{p^{*} x}\right) \leq d\left(\gamma_{p x} 0, \gamma_{p^{*} x}\right)=d\left(p, \gamma_{p^{* x}}\right) \\
& \leq d\left(p, p^{*}\right)=d\left(p, B_{r}\right) .
\end{aligned}
$$

Now by proposition 4.3 it follows that $y=\gamma_{p x}(-\infty) \in S_{1}$. Using the argument above we obtain

$$
d\left(\gamma_{p x}(-t), B_{r}\right)=d\left(\gamma_{p y} t, B_{r}\right) \leq d\left(p, B_{r}\right) \quad \text { for all } t \geq 0 .
$$

The work above shows that

$$
h(t)=d\left(\gamma_{p x} t, B_{r}\right) \leq d\left(p, B_{r}\right)=h(0) \quad \text { for every } t \in \mathbb{R} .
$$

The convex function $h(t)$ has a global maximum at $t=0$, which implies that

$$
h(t)=d\left(\gamma_{p x} t, B_{r}\right) \equiv c>0 .
$$

Therefore every inequality in the paragraph above becomes an equality. From the first inequality it follows that for every $t$ the foot of $\gamma_{p x} t$ on $B_{r}$ actually lies on $\gamma_{p^{*} x}$. Hence

$$
P\left(\gamma_{p x} t\right)=Q\left(\gamma_{p x} t\right) \quad \text { for every } t \in \mathbb{R},
$$

where $Q: H \rightarrow \gamma_{p^{*} x}(\mathbb{R})$ is the orthogonal projection. It now follows that

$$
d\left(\gamma_{p x} t, B_{r}\right)=d\left(\gamma_{p x} t, \gamma_{p^{*} x}\right) \equiv c>0 \quad \text { for every } t \in \mathbb{R},
$$

and hence $\gamma_{p^{*} x}(-\infty)=\gamma_{p x}(-\infty)=y$. By $\left[8\right.$, prop. 5.1] we conclude that $\gamma_{p x}$ and $\gamma_{p^{*} x}$ bound a flat strip in $H$. The inequalities of the previous paragraph also show that $d\left(p, \gamma_{p^{*} x}\right)=d\left(p, p^{*}\right)$ and hence

$$
Q\left(\gamma_{p x}(0)\right)=Q(p)=p^{*}=\gamma_{p^{*} x}(0) .
$$

From this, and the previous fact that $\gamma_{p x}, \gamma_{p^{*} x}$ bound a flat strip in $H$ it follows that

$$
Q\left(\gamma_{p x} t\right)=\gamma_{p^{*} x} t \quad \text { for every } t \in \mathbb{R} .
$$

Hence $P\left(\gamma_{p x} t\right)=\gamma_{p^{*} x} t$ for every $t$, which proves the second assertion of lemma 5.5a.

Let $q$ be any point of $B_{p}$. We wish to show that $d\left(q, B_{r}\right) \leq d\left(p, B_{r}\right)$. Let $A_{p}$ denote the set union of the geodesics $\gamma_{p x}(\mathbb{R})$ as $x$ ranges over $S_{1}$. Let

$$
X=\left\{q \in B_{p}: d\left(q, B_{r}\right) \leq d\left(p, B_{r}\right)\right\}
$$


The set $X$ is a closed convex subset of $B_{p}$ by the convexity of the function $\xi \rightarrow d\left(\xi, B_{r}\right)$. By the work above we have shown that $A_{p} \subseteq X$ and hence $B_{p} \subseteq X$ since $B_{p}$ is the closed convex hull of $A_{p}$. Therefore $B_{p}=X$, which completes the proof of lemma 5.5a.

Proof of lemma 5.5b. Let $p, q$ be arbitrary points in $\tilde{H}$ such that $B_{p}$ and $B_{q}$ are distinct minimally convex sets. If $\xi \in B_{p}$ then $d\left(\xi, B_{q}\right) \leq d\left(p, B_{q}\right)$ by lemma 5.5a. On the other hand $p \in B_{\xi}$ since $B_{p}$ is minimally convex and hence $d\left(p, B_{q}\right) \leq d\left(\xi, B_{q}\right)$ by lemma $5.5 \mathrm{a}$. It follows that $\xi \rightarrow d\left(\xi, B_{q}\right)$ is constant on $B_{p}$ and similarly $\eta \rightarrow d\left(\eta, B_{p}\right)$ is constant on $B_{q}$.

Let $p^{*} \in B_{p}$ be arbitrary, and let $q^{*} \in B_{q}$ be the foot of the perpendicular from $p^{*}$ to $B_{q}$. Let $p^{* *} \in B_{p}$ be the foot of the perpendicular from $q^{*}$ to $B_{p}$. To complete the proof of lemma $5.5 \mathrm{~b}$ we must show that $p^{*}=p^{* *}$. Observe that

$$
d\left(p^{*}, q^{*}\right)=d\left(p^{*}, B_{q}\right)=d\left(p^{* *}, B_{q}\right) \leq d\left(p^{* *}, q^{*}\right)=d\left(q^{*}, B_{p}\right) \leq d\left(q^{*}, p^{*}\right) .
$$

The second equality comes from the conclusion of the preceding paragraph. Hence all inequalities are equalities and in particular $d\left(q^{*}, p^{* *}\right)=d\left(q^{*}, p^{*}\right)$. Therefore $p^{*}=p^{* *}$, since there is a unique point in $B_{p}$ closest to $q^{*}$. The proof of lemma $5.5 \mathrm{~b}$ is complete.

This work was supported in part by NSF Grant MCS-7901730.

\section{REFERENCES}

[1] R. Bishop \& B. O'Neill. Manifolds of negative curvature. Trans. Amer. Math. Soc. 145 (1969), 1-49.

[2] S. Chen \& P. Eberlein. Isometry groups of simply connected manifolds of nonpositive curvature. Illinois J. Math. 24(1) (1980), 73-103.

[3] P. Eberlein. Geodesic flows on negatively curved manifolds II. Trans. Amer. Math. Soc. 178 (1973), $57-82$.

[4] P. Eberlein. Geodesics and ends in certain surfaces without conjugate points. Memoirs Amer. Math. Soc. 13(2), Number 199, March 1978.

[5] P. Eberlein. Lattices in spaces of non-positive curvature. Annals of Math. 111 (1980), 435-476.

[6] P. Eberlein. Isometry groups of simply connected manifolds of non-positive curvature II. Acta Math. 149: 1-2 (1983), 41-69.

[7] P. Eberlein. Geodesic rigidity in compact nonpositively curved manifolds. Trans. Amer. Marh. Soc. 268 (1981), 411-443.

[8] P. Eberlein \& B. O'Neill. Visibility manifolds. Pacific J. Math. 46 (1973), 45-109.

[9] L. W. Green. Surfaces without conjugate points. Trans. Amer. Math. Soc. 76 (1954), 529-546.

[10] D. Gromoll \& J. Wolf. Some relations between the metric structure and the algebraic structure of the fundamental group in manifolds of non-positive curvature. Bull. Amer. Math. Soc. 77 (1971), $545-552$.

[11] M. Gromov. Lectures at College de France. Spring 1981.

[12] E. Heintze \& H. C. Im Hof. Geometry of horospheres. J. Diff. Geom. 12(4) (1977), 481-491.

[13] S. Helgason. Differential Geometry and Symmetric Spaces. Academic Press: New York, 1962.

[14] F. I. Karpelevic. The geometry of geodesics and the eigenfunctions of the Beltrami-Laplace operator on symmetric spaces. Trans. Moscow Math. Soc. (AMS translation) Tom 14 (1965), 51-199.

[15] S. Kobayashi \& K. Nomizu. Foundations of Differential Geometry, Vol. 1, pp. 179-193. J. Wiley and Sons: New York, 1963.

[16] H. B. Lawson \& S.-T. Yau. Compact manifolds of non-positive curvature. J. Diff. Geom. 7 (1972), 211-228. 
[17] G. D. Mostow. Strong Rigidity of Locally Symmetric Spaces, Annals of Math. Studies number 78. Princeton University Press: Princeton, New Jersey, 1973.

[18] J. Wolf. Homogeneity and bounded isometries in manifolds of negative curvature. Illinois J. Math. 8 (1964), 14-18.

[19] J. Wolf. Spaces of Constant Curvature, 2nd edition. Published by the author: Berkeley, California, 1972. 